"Marijuana Moms":

The collective work of negotiating individual and group identity in the age of cannabis legalization

A Dissertation
presented to
the Faculty of the Graduate School
at the University of Missouri-Columbia
In Partial Fulfillment
Of the Requirements for the Degree
Doctor of Philosophy
by
JENNIFER DAWN WILSON
Dr. Wayne Brekhus, Dissertation Advisor
MAY 2019


(C) Copyright by Jennifer Dawn Wilson 2019

All Rights Reserved 
The undersigned, appointed by the dean of the Graduate School, have examined the Dissertation entitled

\section{"Marijuana Moms": The collective work of negotiating individual and group identity in the age of cannabis legalization}

presented by Jennifer Dawn Wilson a candidate for the degree of Doctor of Philosophy in Sociology and hereby certify that, in their opinion, it is worthy of acceptance.

\begin{tabular}{l}
\hline Professor Wayne Brekhus \\
\hline Professor Victoria Johnson \\
\hline Professor Rebecca Scott \\
\hline
\end{tabular}

Professor M. Heather Carver 


\section{DEDICATION}

To Jade Huntington (May 30, 2001- November 24, 2013)

The love of my life, my inspiration, my angel. We did it, Baby girl.

"You are the sun, the moon, and the stars"

I could not have accomplished this without the love and support of my boys; James, Jet, Cole, and Rivers. Thank you for all of the love and support that you have shown as I have struggled and succeeded during my time in graduate school. You all are my reason for breathing, for moving forward, for never giving up. I will always acknowledge this work and all the work it leads to in the future as products not of my individual efforts but rather the collective work of a family dedicated to making our dreams come true, I love each of you more than I can ever express.

On to the next chapter called LIFE! 


\section{ACKNOWLEDGEMENTS}

Dr. Wayne Brekhus, my Advisor and Chair. Thank you for all of the insight, encouragement, and patience through what were undoubtedly the most difficult years of my life. I am forever grateful that you continued to recognize my talent, my passion, and my ability throughout this entire process. You helped me to see the practical value in the work that I do and to love the classroom like it is my second home.

Dr. Victoria Johnson, thank you for constantly challenging me to think harder on all of my projects and to push past the comfortable answer. You have remained a constant reminder in my head that our work is important and to be taken seriously.

Dr. Becky Scott, I am forever grateful for your insight on this project and your extremely helpful feedback that kept me constantly thinking about how my work fit into larger discourses in our discipline. I am sure that your advice will serve me well as I continue to develop my research goals.

Dr. Heather Carver, thank you for all of the ways you supported me through this process. You were an excellent resource for considering all of the ways that gender and gender roles were being performed at all times.

Dr. Ed Brent, thank you for all of the support you have shown to me and my family. I will miss my time at the University of Missouri-Columbia and the family that I gained over the last ten years. Thank you for believing in me and supporting me through the hardest years of my life. 


\section{TABLE OF CONTENTS}

ACKNOWLEDGEMENTS . $\mathrm{V}$

ABSTRACT. ix

CHAPTER ONE: THE STUDY 1

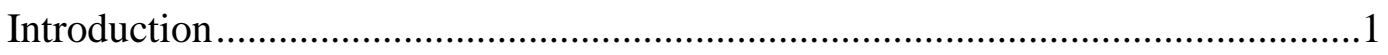

Legalization in the United States ....................................................................6

Who is really using marijuana in America?..........................................................

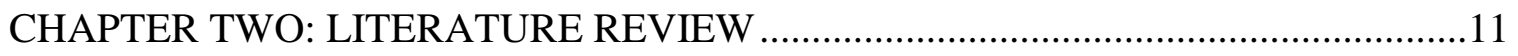

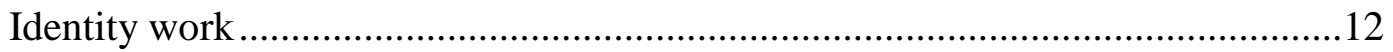

Greedy institutions and identities......................................................................

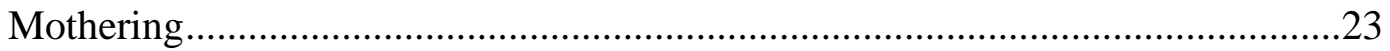

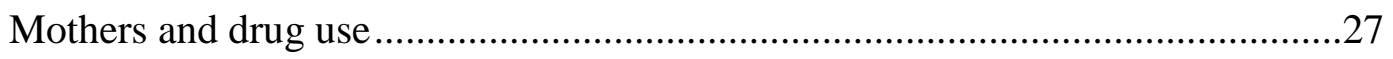

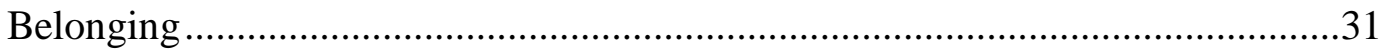

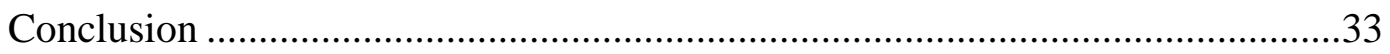

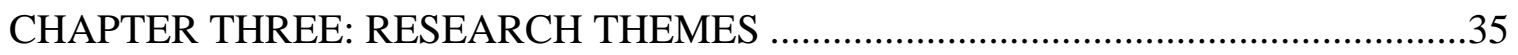

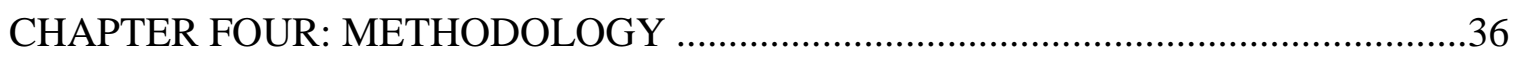

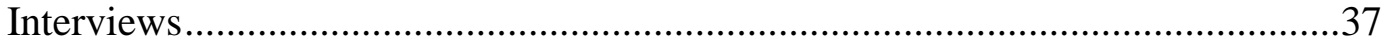

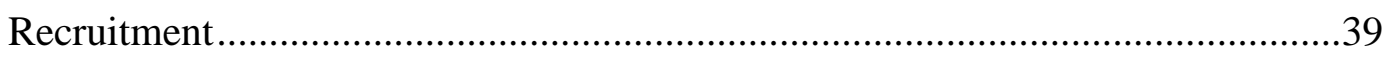

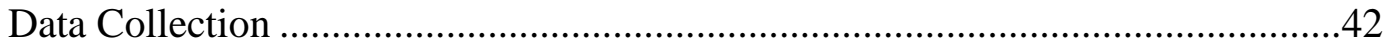

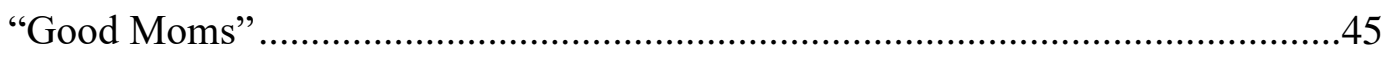

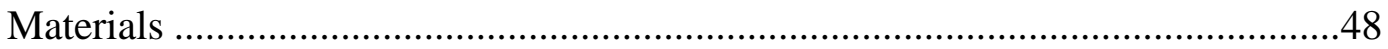

CHAPTER FIVE: Motherhood- the greediest identity.................................................50

The greedy identity of motherhood......................................................................50 
Internalizing the cultural narrative.

"Self-sacrificing moms are great".....................................................57

“Good moms are present \& sober, right? ”.........................................59

"She loves and accepts her children" ..................................................61

"Always there to help and support "..................................................62

Counternarratives of motherhood ….............................................................64

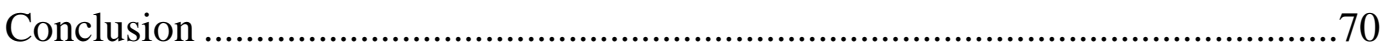

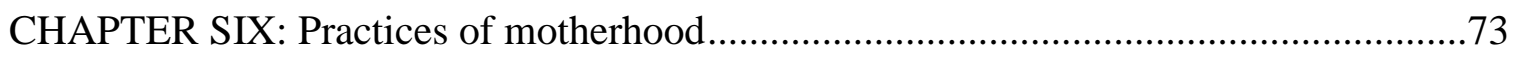

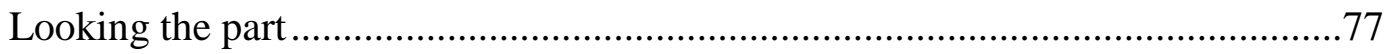

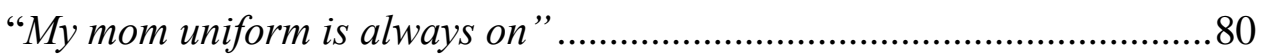

"My kids are clean and dressed to impress ".........................................82

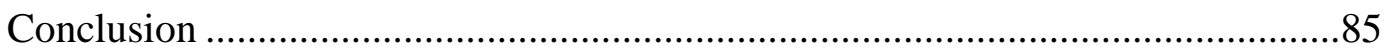

CHAPTER SEVEN: "I can be an amazing mom and be high": managing incompatible

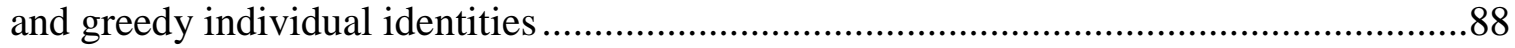

Personal Counternarratives ...................................................................91

Holding a competing greedy identity- Marijuana user .....................................94

"Mother's little helper" ...................................................................96

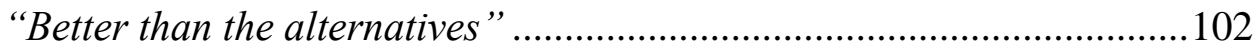

"Responsible use is key" ................................................................. 104

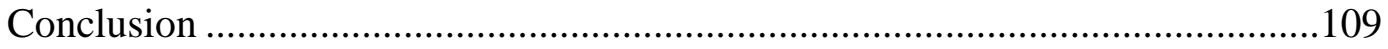

CHAPTER EIGHT: The groupwork of reconstructing the collective identity of "good mom”.

Selective self-presentation

The Cage: Online groups as identity generating spaces .......................118

Finding your Tribe: Belonging and meaning making practices .............122

Conclusion 
CHAPTER NINE: DISCUSSION/CONCLUSION

Changing cultural meanings one post at a time ................................................130

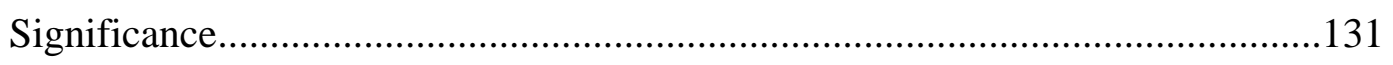

Implications for future research .................................................................134

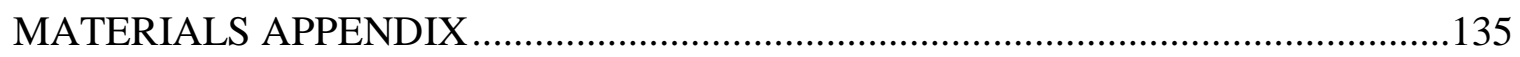

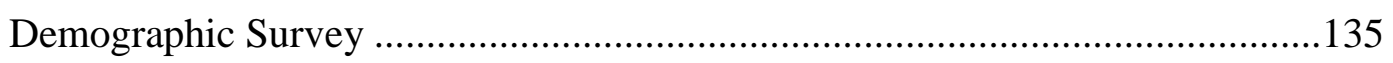

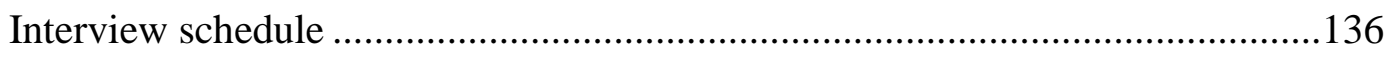

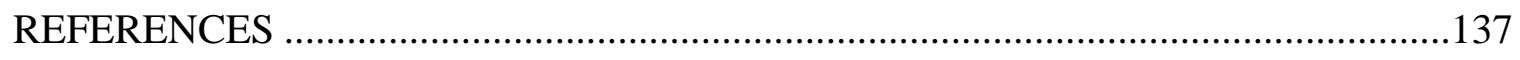

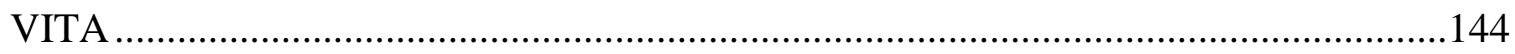




\begin{abstract}
$\underline{\text { Abstract: }}$
This research is the result of a qualitative study that explored the ways in which marijuana using mothers come to identify as such and how they structure their relationships and parenting as a result. The experiences of 57 self-identified marijuana using mothers (aged 20-48 years-old) from across the United States participated in semi-structured interviews and shared their everyday experiences with both marijuana use and motherhood. Participants were all mothers with children between 3 months and 19 years at the time of the interviews. A thematic narrative analysis uncovered common experiences among these women in constructing both individual and group identity: Participants varied in how each of these themes identified were reflected in their lives, depending upon each participant's interpretation of her local social context. Both motherhood and self-identifying as a marijuana user were valuable and meaningful parts of their identity
\end{abstract}




\section{CHAPTER ONE:}

\section{THE STUDY}

\section{Introduction}

In an anonymous blog a mom writes:

“As suburban moms go, you can't get any more typical than me. I'm active in my kids' lives and in my community, a responsible and trustworthy adult. I'm a good wife, a good friend, a good daughter, and a mother who loves her kids above all else. You would want someone like me to babysit your kids or house or pet sit while you were on vacation.

I have a job, I worry about keeping my family healthy, I like my house tidy, but sometimes that just means Febreeze-ing everything. I wear skinny jeans and cardigans and flats, typical mom wear.

In my purse, I carry my voter ID card, my passport, my library card, my driver's license (20 years behind the wheel and not a blemish on my record, knock on wood), and a bunch of Band-Aids and snacks because you never know where you'll find a kid, or fellow mom, in need.

I dye my hair to cover grays and slather my face with drug store wrinkle cream hoping that it works as well as the expensive kind that I can't afford because I have to feed four growing boys. I start getting tired by 9 p.m., so I'm no wild partier. Once in a while at the end of a particularly long day, I'll relax on my couch with a glass of wine or two.

This is me, and I'm probably a lot like you. I could be your neighbor. I could be your friend. We could hang. There's just one thing: Go back to that last sentence and change "relax on my couch with a glass of wine or two" to "relax on my couch with a joint."

When considering the cultural narrative that surrounds motherhood in the United

States it is clear that the white, middle-class standard set by 1950's and 1960's television sitcoms like, Leave it to Beaver continues to be the primary representation called upon when discussing 'good moms'. Claire Dunphy, of Modern Family a long running television sitcom, can be seen as the new symbol of motherhood as she embodies the characteristics established by June Cleaver while also representing the more modern ideal 
that allows working-moms to hold tight to this coveted identity of being a 'good mom'. Despite this loosening of the cultural expectations surrounding some types of work and motherhood the cultural symbols used to define a "good mother" continue to be defined by this standard for mothers of all racial, socio-economic, and marital statuses.

This understanding of a 'good mom' as someone who is also suitably-aged (midtwenties to mid-thirties seems to be the standard), white, western (American or European), and middle-class has become hegemonic and so pervasive in American culture that a large number of women accept and embody it without question (Arendell 1999; Lupton and Fenwick 2001; Phoenix and Woollett 1991). However, there is research that suggests that this pressure to fit the hegemonic model is not experienced equally by all mothers. For example, mothers in racialized groups and from lower socioeconomic class groups do not experience the social pressure to fulfill the role of intensive mother with the same pressure as white, middle-class mothers (Barnes 2008; Lareau 2003).

As marijuana legalization takes hold in the United States, mothers are increasingly becoming the targets of criticism and a rhetoric of shame that threatens a sacred part of their identity, their coveted identities as 'good moms'. Mothers who use illicit drugs, even those that have recently been legalized like marijuana, find themselves outside of the hegemonic model of what society considers a "good mother" and the subjects of often overt social shaming (Wiegers and Chunn 2015). Frequently concerns are voiced about potential harms to their children and their inability to "be good moms" due to their drug use, often regardless of frequency or other consumption practices (Banwell and Bammer 2006). 
This is primarily because the dominant cultural narrative surrounding motherhood in the United States is often one that is saturated with ideas and beliefs that follow the ideology of "intensive mothering". Telling mothers of all socio-cultural backgrounds that in order to live up to society's standard of being a 'good mom' you must have a practice of mothering that is "child-centered, expert-guided, emotionally absorbing, labor intensive and financially exhaustive" (Hays 1998; Blair-Loy 2003; Stone 2007; McQuillan, Greir, Shreffler, and Tichenor 2008). This style of mothering has long been dominated by middle-class women and is an essential practice that allows middle-class values to be reproduced in the family. This style of mothering is also particularly difficult for mothers who are not White, not married, and not middle-class to achieve (Lareau 2003; Edin and Kefalas 2005; Banwell and Bammer 2006; Dodsworth 2012; Elliot, Powell, and Brenton 2013; Wiegers and Chunn 2015).

The increasing pressure for mothers to fulfill their role as an intensive mother while also defining herself as a woman outside of her maternal duties has highlighted the contradictory forces shaping the lives and identities of mothers in modern western society (Hays 1996, Collett 2005). Mothers who hold an identity outside of this understanding or engage in behaviors seen as "not for moms" (i.e. drug use, inappropriate attire, time intensive hobbies, or traveling careers...) find themselves in jeopardy of being constructed as part of an "at-risk group" often defined as "sick or deviant" (Banwell and Bammer 2006).

This dominant cultural narrative regarding mothers' drug use is another way that we see dominant middle-class values reproduced through social understandings of motherhood. These middle-class ideologies work together to construct drug-using 
mothers as "doubly deviant" (Broom 1994). These mothers may go to great efforts to maintain their hold on the 'good mom' identity. Most mothers who engage in drug use are fully aware that this conflicting part of their identity threatens to eradicate the coveted status of 'good mom' (Banwell and Bammer 2006). This makes their identity as "marijuana user" a "greedy" and dangerous one that must be carefully negotiated (Brekhus 2003).

Most of the research focused on drug use and identity has centered on men and their drug usage patterns. As a result, the experiences of women were largely invisible from academic discussions regarding drug using identities until about forty years ago. This is not to say that women were not engaging in drug use, just that their experiences were yet to be included in the academic and policy related discussions being had. If women's experiences were included in the academic research, they were often studied in abstracted ways that rarely reflected their "real" experiences. Consequently, women who use drugs have been constructed in ways that are quite different from the construction of men who engaged in use of the same drugs. Women are often characterized as deviant, or as engaging in risky behavior. This is only amplified by the co-identity marker of mother. Mothers who engage in drug use often receive the harshest criticisms and as a result are quickly defined as "bad moms".

This disproportionate stigmatization of mothers who use drugs is not confined to public opinion, it is apparent that social science research has contributed to and reproduced the cultural narratives that have erased women's lived experiences as well. One of the most important ways we see the consequences of this is the lack of mothers willing to openly discuss their drug use. Women with children are more likely to engage 
in drug use in private settings or alone than women without children. Another consequence is that drug-using mothers are motivated by fear of both informal and formal social sanctions for openly discussing their drug-use. Both characteristics of mother's drug use makes it difficult for social scientists to study. But with marijuana legalization spreading, more and more women are becoming open about their use of the drug allowing researchers to create a better understanding of how and why mothers are using marijuana.

Since the legalization of recreational marijuana in the United States began in the mid 1990's, a growing number of mothers (like the one in the opening of this chapter) have become increasingly more willing to be open about their often-stigmatized identity, “marijuana-using mom”. This openness has increased despite a continued stigmatization of this behavior for women, in general, and even more intensely for mothers. Recent studies confirm that the majority of recreational marijuana users are still men, making up approximately $63 \%$ of all users in a 2018 poll $^{1}$. Drug culture and drug use remain maledominated, but women are increasingly joining the ranks of recreational tokers with a growing number of those women reporting to also be mothers. A recent poll found that of the respondents who reported using marijuana regularly $54 \%$ were parents ${ }^{2}$.

However, this growing openness about marijuana use comes at a disproportionate social cost to many groups in society. Minorities, parents, and mothers are all subject to a seemingly different cultural standard with regards to marijuana use. A recent Yahoo News poll asked respondents if "they would lose respect for someone who used

\footnotetext{
${ }^{1}$ https://kdvr.com/2017/04/17/study-majority-of-americans-who-say-they-use-marijuana-are-parents/

${ }^{2}$ http://www.pewresearch.org/fact-tank/2018/10/08/americans-support-marijuana-legalization/
} 
marijuana". About one-third or $31 \%$ of the respondents reported that marijuana use would result in a loss of respect to some degree. When asked if "they would lose respect for someone who used marijuana if they were a parent" an overwhelming $79 \%$ of the respondents answered "yes, they would lose respect for someone who used if they were a parent"3, showing the weight carried by the identity of parent. Many parents who report using the drug believe the social perception of the drug and it's use among adults is "grossly misrepresented" and that false impression is the reason for much of the stigma associated with parents who use the drug, and in particular mothers who use the drug.

\section{Legalization in the United States}

The United States is currently experiencing a period of dramatic shift in the cultural and legal understandings of marijuana, a drug still considered as harmful as heroin by federal standards ${ }^{4}$. By the end of the year 2018 , there were $33^{5}$ states in the U.S. that had adopted legislation supporting medical marijuana usage and sales; with 10 of those 33 states $^{6}$ also adopted legislation supporting recreational marijuana usage and sales. Experts in the field predict that 2019 will bring another half dozen or more states into the mix with the passage of medical and/or recreational legislation following more

\footnotetext{
${ }^{3}$ http://www.pewresearch.org/fact-tank/2018/10/08/americans-support-marijuana-legalization/

${ }^{4}$ Marijuana is current classified as a Schedule 1 Narcotic according to the Federal Drug Administration and the Department of Drug Enforcement. State laws ignore this designation.

${ }^{5}$ States with Legal Medical Marijuana: Washington, Oregon, California, Nevada, Arizona, Utah, New Mexico, Colorado, Montana, North Dakota, Minnesota, Michigan, Illinois, Missouri, Arkansas, Oklahoma, Louisiana, Florida, Ohio, West Virginia, Pennsylvania, New York, Vermont, New Hampshire, Rhode Island, Massachusetts, Connecticut, and Maine.

${ }^{6}$ States with Legal Recreational Marijuana: Washington, Oregon, California, Nevada, Colorado, Michigan, D.C., Vermont, Massachusetts, and Maine.
} 
favorable governors and legislative bodies post the 2018 election cycle ${ }^{7}$. With each new State's legalization efforts, we see the national attitude towards marijuana as a medicinal and a recreational drug shift. A recent poll reported that approximately $63 \%$ of Americans over the age of 18 believe that marijuana should be made legal, a stark increase from the just $31 \%$ who supported legalization $2000^{8}$.

The United States has a long, sorted, and well-documented history with marijuana. The drug has historically been associated with and used to stigmatize and criminalize racial minorities (Glasser 1999; Pager 2001; Laguna 2007). However, over the last three decades America's attitude regarding the drug has slowly been evolving. From the Reefer Madness hysteria of 1936 to the first Medical Marijuana initiative passed in the city of San Francisco in 1991, the majority of Americans have gradually come to accept Marijuana ${ }^{9}$. California became the first state to legalize the use of marijuana for medicinal purposes in 1996 and over the last 22 years another 32 states have followed suit passing legislation the decriminalizes the use, possession, and/or cultivation of the drug in varying degrees. The growing acceptance of marijuana use for medicinal purposes played no small part in the 2012 passing of Proposition 64, making Colorado the first state to legalize the recreational use of marijuana for it's over the age of 18 citizens in January of 2014. Colorado then paved the way for 9 more states to decriminalize the recreational use of marijuana by 2018, opening a billion-dollar industry and the minds of millions of Americans. The legalization of the recreational use of

\footnotetext{
${ }^{7}$ https://www.forbes.com/sites/tomangell/2018/12/26/these-states-are-most-likely-to-legalizemarijuana-in-2019/\#11c711465add

${ }^{8}$ http://www.pewresearch.org/fact-tank/2018/10/08/americans-support-marijuana-legalization/

${ }^{9}$ http://www.pewresearch.org/fact-tank/2018/10/08/americans-support-marijuana-legalization/
} 
marijuana has led to a new openness and acceptance of its use that has shifted the demographics of the open or "out user" dramatically.

\section{Who is really using marijuana in America?}

A poll taken in October of 2017 revealed that roughly 64\% of Americans support the legalization of marijuana, at least for medicinal purposes. That same poll revealed that $54 \%$ of those who reported using the drug more than twice in the last year were also parents ${ }^{10}$. Recent studies, like these, demonstrate that marijuana is no longer the drug of misguided youths, the poor, or racial minorities as many once believed. Rather, it is increasingly becoming popular amongst middle-class, White parents.

The ongoing change in legal status and state policy has opened the "pot market" to suburban, middle-class parents due to its increased accessibility and the growing variety of products available for the new user's consumption. This new-found accessibility and social openness allow suburban moms to trade in their wine glasses for bongs, pipes, vape pens, edibles, and THC infused drinks and creams. At the same time, this growing marketplace has contributed to a cultural shift that has rippling effects through countless social practices, roles, obligations, experiences, expectations, and understandings of the drug itself, its effects, its use, and the way we understand those who use marijuana.

${ }^{10}$ https://kdvr.com/2017/04/17/study-majority-of-americans-who-say-they-use-marijuana-are-parents/ 
It is this new demographic that is the focus of this study. They are what 'good moms' are supposed to be in every way, except that they are high. They sit in privileged positions at the top of a social ladder of mothers. At first glance, they fit the cultural narrative about what a 'good mom' looks like and does. It is this lack of stigmatized identities that makes them ideal for uncovering the degree to which drug use affects these mothers' identities and the strategies they employ to protect their valued status of motherhood from the greedy implications of this competing identity marker.

The increasing number of states that have passed legislation making both medical and recreational use of marijuana legal not only signal a shift in public perception of the effects of the drug but also those who use the drug. My goal is to provide a more nuanced understanding of the lives of women who are mothers and marijuana users in the era of legalization in an attempt to give voice to their parenting beliefs, experiences, and practices but also to theorize the tensions and contradictions surrounding the ideology of intensive mothering.

My hope is that my research will help to develop a better understanding of how women, and mothers, in particular, are negotiating the shifting cultural terrain in efforts to construct new cultural understandings of these competing identities. The narratives that result from this research have the potential to be powerful meaning making tools and invaluable resources in shaping social and cultural understandings of marijuana using mothers and their ability to be 'good moms'. By exploring the narratives of mothers who use cannabis to uncover their experiences of stigma, social sanctioning, and acceptance we can examine the meaning making and collective work that is being done by these 
women to change cultural perceptions of mothers who use marijuana as legalization becomes more widespread in the United States.

How do women with children, who fit the hegemonic conception of 'good mom' in seemingly every way, negotiate this greedy identity when it runs counter to the cultural narrative surrounding their consumption of marijuana? 


\section{CHAPTER TWO:}

\section{LITERATURE REVIEW}

The literature that shapes the analytical framework for this research is drawn from two main bodies of scholarship, respectively.

The first, the body of Symbolic Interactionist and Social Construction of identity literature exploring stigma and identity that has amassed since Erving Goffman published his pioneering work, Stigma. For Goffman (1963) stigma is the negative labeling of what is perceived to be "a deeply discrediting attribute". An attribute that can distinguish its bearer from the "normal" others within the group or society. Goffman identified three types of attributes that could be seen as attracting stigma- a physical deformity, an individual character flaw, and a "tribal stigma of race, nation, and religion" (1963: 3-5). I follow the broadened conceptualization of stigma developed by Link and Phelan (2001) that emphasizes the relationships among labeling, stereotyping, status loss, discrimination, and social exclusion.

The second, is the expansive feminist literature on motherhood that has proliferated since the 1970's. This literature has exposed and challenged oppressive constructions of motherhood for decades (Warrior 1968; Rich 1977; Boulton 1983; Hays 1996; Bailey 1999; Arendell 2000; Bock 2000; Bailey 2001; Lupton and Fenwick 2001; Blair-Loy 2003; Edin and Kefalas 2005; Collins 2005; Collett 2005; Stone 2007; Whitley and Kirmayer 2008; Chesley 2011; Ellis-Sloan 2013; Ennis 2014; Zartler 2014; Elliott, Powell, and Brenton 2015). For some the feminist perspective's practice of challenging traditional norms and ways of life has played a primary role in the cultural shifts we see 
in society, our institutions, and our identities (Beck 1992). Despite criticisms feminist theorists have continually taken on critical examinations of important social institutions in efforts to reduce inequality; such as the family, the labor market, medicine, the law, and education.

Both bodies of literature are invaluable in my analysis of the individual and collective work that goes into shifting the cultural narrative surrounding motherhood by mothers who seemingly fit the hegemonic model of "ideal mother", except for the fact that they use marijuana.

\section{Identity work}

Identity work is defined as, "anything people do, individually or collectively, to give meaning to themselves or others" (Schwalbe and Schrock 1996:115). There are two levels of "work" that are inherent in this type of analysis. The first is the communal or "the creation of identities as widely understood signs with a set of rules and conventions for their use". The second is the individual or the "use of these signs, rules, and conventions by individuals to create images of themselves" (Schwalbe and Schrock 1996:115). Snow and Anderson's (1987) study of the homeless and their use of narrative to construct their identities becomes vital in helping us to understand how what we call "identity talk" can be theorized as adaptive responses to the material conditions of people's lives. This same "identity talk" can also be employed to reshape the social meanings attached to an entire group, it is in this case that it is a tool used to redefine the cultural meanings of what it is to be a 'good mom' (1342). 
This research draws heavily on the theoretical influences of classical Symbolic Interaction theories, Social Constructionism, and feminist theories of motherhood. In many ways it parallels the work of Giddens (1992) and Beck (1992). This study attempts to uncover how a 'risk society' (Beck 1992) emerges through the dissolution of traditional norms and ways of life. How are identities in a 'risk society' are cultivated? This study hopes to uncover the way that Individuals actively engage in making their own biographies, rather than just being passive recipients of society, its expectations, and meanings. This assertion of autonomy and agency in identity formation echoes the work of Gecas and Schwalbe (1983) and points to the ways that "self-concept development are issues of autonomy and efficacy"; where autonomy refers to the ability to choose one's own course of action, and efficacy to the belief in self-as-cause" (81).

One of the key tenants of Symbolic Interaction theory is that identities are socially constructed (Gubrium and Holstein, 1990). According to this theoretical perspective, the self is shaped by countless messages from our surroundings, the media, and a host of significant others who inhabit our social worlds (Goffman 1959). Often the representations of mothers that the media constructs may lead people to believe that all mothers "are autonomous and efficacious in the sense that they make choices regarding how to parent and that their children are the product of such choices. It is important to realize, however, that autonomy and efficacy, particularly in motherhood, are disproportionately luxuries of the middle and upper classes" (Collett 2005: 328-329). Sharon Hays (1996) argues that social class is key to the ideology of intensive mothering. Mothers who occupy middle- and upper-class positions certainly find "giving unselfishly of one's time, money, and love to one's children" a less challenging social expectation 
than mothers who occupy lower social class positions and often lack the financial resources to live up to this social expectation (Collett 2005: 329).

Due to the social component that exists in all identity development, a social actor may lay claim to an identity, such as 'good mom'; however, the validity of that claim will depend on the responses of significant others in the actor's social world (Gubrium and Holstein 1990). The work of Susan Douglas and Meredith Michaels (2004) describes what they call a "New Momism" for the modern mother. It insists that for today's moms "to be a remotely decent mother, a woman has to devote her entire physical, emotional, and intellectual being, 24/7, to her children" and she is supposed to "enjoy every minute of it" (4). We see this cultural narrative recreated again and again in parenting books, magazines, value-based marketing, and the media coverage of celebrity mothers.

In their aptly entitled work, The Mommy Myth: The Idealization of Motherhood and How it has Undermined Women, Douglas and Michaels (2004) argue that this new ideology surrounding motherhood is "an equal opportunity oppressor, affecting both stayat-home and working mothers. The work of a few feminist researchers supports the claim that the portrayal of working mothers in the media and by experts as neglectful or as a threat to their children's emotional development. As a result, it is argued that working mothers become desperate to show that they are supermoms that can manage to work outside of the home and still raise happy, healthy children and also maintain blissfully happy marriages (Eyer 1996, Hays 1996, Chesley 2011, Stone 2007, Douglas and Michaels 2004; Blair-Loy 2003).

This is not to say that mothers who do not work outside of the home do not face pressures of their own. Hays (1996) argued that as the ideology of intensive mothering 
becomes the normative standard for all mothers the centrality of this identity becomes more apparent. Women who stay at home with their children often report that they "lose their personal identities and motherhood monopolizes their time" because of this they often "derive their sense of meaning and purpose from their children and their role as mothers" (Boulton 1983)

According to social constructionists, "motherhood" can be seen as the product of individual, social, and cultural discourses which interact to create particular meanings concerning mother identity (McGannon and Schinke 2013). From this perspective, "discourses circulate certain meanings about motherhood which become forms of truth and difficult to challenge because they are also tied to gender ideologies (i.e., expected behaviours based on cultural values and norms). These practices include the prevailing notion that women's true calling is to have children and care for them, and that men's true calling is to be providers" (Bailey 2001).

It is culture that tells us what it means to be a mother. Culture provides a narrative that defines what are appropriate behaviors for mothers. The narrative provided to mothers is one that places extraordinary emphasis on success. This characteristic of motherhood is why it is often perceived as "a central identity issue for many women" (Collett 2005). It also defines for us how motherhood "should" shape your identity (Bailey 2001). Discourses of the 'good mom' are often tied to gender ideologies and constraining caregiving practices in ways that restrict the behaviors that mothers can engage in. Together this points to the way that "social identities are inherently defined by roles and relationships; the identity of mother is distinctive" (Collett 2005). Simply put, being someone's mother (biological or adoptive) is not enough anymore. In today's 
world a mother's success and hold on the identity of 'good mom' is measured not by her practice but "by her child's life and achievement" (Collett 2005:329).

This view of identity as a "joint accomplishment" has long been a key component in the work of social psychologists (Strauss 1959) and symbolic interactionists (Blumer 1969), as well as dramaturgical perspectives (Goffman 1959). By “joint accomplishment” it is argued that identities are only formed when others acknowledge them in you (Goffman 1959; Becker 1953). In this perspective, children become props on the stage of life that mothers can deploy to affirm their goodness, success, and "worth as a human being" (Tardy 2000:444). Conceptualizing identity in this tradition allows us to examine how identities are constructed, negotiated, and repaired through group interactions (Snow and Anderson 1987) and storytelling (Young 1989). While the efforts of individuals to manage or refuse to internalize a stigmatized identity are interesting and important to my analysis, I also argue that it would be shortsighted to neglect the group work that also goes into the identity management of these mothers.

\section{Greedy Institutions and identities}

Feminist scholars have created a wealth of knowledge regarding the ways that Institutions play a significant role in shaping our individual identities (Blair-Loy 2003; Hey 2004, Edin and Kefalas 2005, Stone 2007; Lareau 2011; Mears 2011). This work has revealed that the power of institutions to shape individual identities is often relative to the significance of the intensity with which the individual holds a particular institutional 
status. For example, the degree to which a working-mother identifies with her occupational status. A woman who holds a powerful or professional position prior to becoming a mother may experience greater role conflict in regard to her ability to live up to the "ideal worker" ideology that dominates many of the institutions in which these high profile careers exists than, for example, a mother who enters the workforce after becoming a mother (Blair-Loy 2003; Stone 2007). Brekhus (2003:45) characterizes these as "greedy institutions" as a result of the way these institutions "pervade all facets of members' lives and demand undivided commitment".

Women in today's world are experiencing ever-growing pressures to define themselves by their non-maternal activities. At the same time, the expectations of motherhood and what it means to be a 'good mom' have increased. 'Good mothers' are now seen as those who devote unlimited amounts of time, patience, love, and labor to their children in addition to being narrowly defined as white, appropriately aged, middleclass, and married (Warrior 1968, Boulton 1983, Brown, Small, and Lumley 1997, Lupton and Fenwick 2001). Despite the growing rhetoric about the declining significance of motherhood due to the women's movement "successes", studies have not been able to support such claims (McQuillan, Greil, Shreffler, and Tichenor 2008).

Stone (2007) argues in her influential work, Opting Out: Why Women Really Quit Careers and Head Home, that women experience disproportionate pressure as the result of the institutional practices and expectations placed on them by their conflicting roles of Mother and Professional (Blair-Loy 2003; Stone 2007; Chesley 2011). This is both the effect of the cultural expectations placed on the individual identity as it is the result of the institutional practices of both motherhood and professional employment that 
have become pervasive to the extent that they infiltrate the practices of other institutional roles. For many in professional occupations there is an expectation that an "ideal worker" is always available and accessible to their employer, there is an expectation that the worker will be performing mental labor pertaining to their occupation when away from the work place, and that they will make efforts to tailor their life and daily practices in ways that benefit their employer (Stone 2007). In direct conflict to that is the social expectation that 'good moms' are mothers that are "“'child-centered, expert-guided, emotionally absorbing, labor intensive and financially exhaustive" (Hays 1998; McQuillan, Greir, Shreffler, and Tichenor 2008) in their practice.

For mothers, this infiltration of workplace in their home life becomes particularly problematic due to the pervasive nature of the cultural practices and expectations attached to the role of Mother (Hays 1996; Blair-Loy 2003; Stone 2007; Lareau 2011). I argue that motherhood has become an increasingly "greedy identity". The "greedy identity is a parallel concept, an identity that commands an uncompromising commitment, undiluted by any attributes that could undermine it or by any time commitments that could divide it...Greedy identities require both a full-time and maximum intensity commitment, and this combination of duration and density gives them their greedy quality of dominance" (Brekhus 2003:45).

It is theorized that despite the often restrictive, time-consuming, and financially exhaustive practices associated with being a 'good mom' in U.S. (and increasingly European) culture that mothers of all socioeconomic statuses and racial/ethnic backgrounds have internalized the narrative of "intensive mothering" to varying degrees. It appears that mothers in each of these groups define 'good moms' as those who manage 
to achieve the cultural expectations of "intensive mothering" and likewise judge their own quality of mothering by the standard embedded in them (Blair-Loy 2003; Hey 2004; Stone 2007; Chesley 2011).

It is no secret that all social identities are not equal. Some social identities have little social cost to the individuals that possess them, while others can carry devastating social costs for their possessors. Identity markers that have low social costs include identities connected to hobbies and cultural tastes and tend to carry lower social costs than identity markers such as racial identity, political affiliation, gender, sexuality, and religion (Brekhus, 2003). A person may identify as a "coffee lover", an "animal lover", a hunter or a vegan with very little social cost to them personally. There are few, if any, social sanctions for carrying any of these identities. It is highly unlikely that any of these low-cost identity markers would come with social consequences to the individual who possess them.

In this work I will focus on high-cost social identity markers. Goffman (1963) theorized these high-cost identities as "stigmatized identities", or identities that carried negative social meanings. For him these identities could carry varying degrees of stigma due to the intensity with which society held these negative meanings toward that particular identity status. In some cases, the social cost could be so great to the individual that the stigmatized identity, whether ascribed or achieved, becomes the most significant status a person holds in a given social context. Goffman referred to these statuses as "Master Statuses", or statuses that can erase or negate other identity markers that an individual may possess. 
Master statuses may include an ascribed attribute like a person's race. In the United States an individual's race can become their master status in a multitude of social contexts. An African-American from a rural Midwest state who pursues a career in country music, for example may find that his race becomes a barrier he must overcome, a part of his identity he must be constantly aware of because it is clearly the motivations behind many of the interactions, he has with white record label executives. Prejudice attitudes like this reflect only one dimension of the power of stigmatizing master statuses. The empirical evidence supporting claims that African-Americans are subjected to disproportionate risks of being brutalized or killed by a police officer is another. Master statuses can also be achieved statuses, like a person's occupation. For example, women who choose professions in the sex industry often find themselves marked as "bad women" or "poor examples" and spend a significant portion of their identity management strategies on overcoming the social stigma that is attached to their profession.

For this work, the concept of Master status is important because of the way the concept parallels the concept of greedy identities, which will become the focus of this work. While Master statuses typically refer to statuses that carry negative social meanings and can erase or even eradicate other social statuses or characteristics, greedy identities are those that permeate and wash over "other aspects of (his) self and pervades all (his) social networks" (Brekhus 2003). For example, a deviant Master status such as Felon may be powerful enough to erase the person's ability to engage with society as s/he once did. A person convicted of a sex crime may find that this quickly becomes their master status. This status can erase any social prestige offered by a variety of other social 
statuses that individual may possess. It may also eliminate a number of highly valued social privileges.

For his research on gay identity, Brekhus (2003) theorized that identity is often experienced in varying intensity and for varying duration resulting in what he calls lifestylers, commuters, and integrators. Lifestylers can be seen as those who live a particular identity marker with high intensity for long periods of time, or high duration. These individuals become their identity markers and live them with great intensity. Those who do not experience "marked identity spaces" in their own lives may however find that they must live this "marked identity" at a lower intensity than those who do have those communities or integrate their marked identity with all of their others. Brekhus calls this group integrators. Others who do not experience "marked identity spaces" may find that they submerge their marked identities at times and at others they live it openly. Brekhus refers to this last group as commuters (46).

A greedy identity is different from a Master status in several ways. The most significant for us is the difference in the effect. While a master status has the ability to erase parts of an identity, a greedy identity is one that works to transform other aspects of an individual's identity. Being a working-mother does not erase a mother's identity or her identity as worker. It only complicates them both. Greedy identities are those that permeate other aspects of your life through institutionalized and culturally accepted practices. In this sense motherhood becomes easily illustrated as a greedy identity. While motherhood may be an important social status for many women and certainly the motivation for many others' interactions with her, it also does not erase those parts of her identity. She still holds them they are just transformed by the other simultaneous statuses 
she holds. I hold that motherhood is a greedy identity that requires the alteration and transformation of the other social identities she may possess.

It is for this reason that it is important that we consider the ways that language is essential to constructing our social realities. This becomes an important way for us to uncover the cultural understandings of these greedy identities. For example, if a woman who is a mother works outside of the home then she is referred to as a "working-mom". Motherhood seems to carry an identity permeating weight that one would be hard pressed to find in another achieved social status.

Fatherhood, for example is far less greedy and becomes a more flexible identity marker in many ways. This too becomes clear by examining the differences in the language we use when we talk about men who are parents and also engage in work outside of the home, as we do not refer to these fathers as "working-dads". Another example would be the differences in the ways we discuss mothers who engage in recreational sports or hobbies versus the language we use when we talk about fathers who engage in similar recreational sports or hobbies. These dads are not stigmatized as "bad dads" because they devote time to endeavors other than their children. Even if their participation means they will be absent from the events of their children, a luxury few mothers enjoy.

This flexibility in the identity of father reduces the tension created by many of the competing social identities men carry. Rarely are fathers seen as "bad dads" for holding competing identities. In fact, their ability to juggle these competing and often contradictory identities has the effect of increasing the positive ways in which society sees them as fathers. 


\section{Mothering}

There are several biographical events that can result in the addition of the label, mother. The events that result in this new identity range from "conceiving, giving birth to, or adopting, a child" (Collett 2005). Each of these biographical events carry social meanings that will "influence, but do not fully determine the construction or assertion of...personal identities" (Snow and Anderson 1987: 1347). It is important to note that not all individuals will internalize or perform this new identity in the same way. "Personal identities are self-designations that arise in the course of interaction, not material things one possesses and displays. In other words, a woman may be become a mother by giving birth, but she truly takes on a mother identity by playing a socially defined, publicly visible role" (Collett 2005).

The history of motherhood in the U.S. is a complicated story that illuminates the cultural narrative used to define 'good moms'. It is clear from looking at this history that WWII played a significant role in shaping that narrative. The war led to a labor shortage that called women out of the home by changing the social conditions and the narrative surrounding female headed households and working mothers. Then, as quickly as it had shifted the narrative about working mothers it sent them back to the home and returning to a rhetoric of shame for mothers who worked outside of the home. Post-WWII women who remained in the workforce or those who joined were effectively erased from public discourse by placing an emphasis on the value of the work that women performed in the home, and the representation of the woman worker as childless (Riley 1983). There is not time in this paper to discuss the history or the evolving cultural narrative surrounding 
motherhood in the United States in detail, but there is no question that the social expectations set out in that changing narrative surrounding motherhood form an important context to the experiences of women (Bailey 1999).

Recent feminist accounts of motherhood have highlighted the problematic nature of the social construction of mothering and the relationship that motherhood has to the oppression of women (Rich 1977, Oakley 1979). The practice of mothering has received increasing attention over the last thirty years in both public and academic discourses highlighting the "contradictory cultural" forces that women in developed countries face in the $21^{\text {st }}$ century (Hays 1996 \& 2003; Stone 2007; Edin and Kefalas 2005; Devault 1999; Collins 1994 \& 2005).

The Women's Movement and its successes in increasing women's participation in the wage labor market, increasing legislation that protects women's rights to reproductive healthcare and choice, increasing protections for women from abuse, and countless other pieces of progressive legislation. All of these have contributed to a cultural narrative about falling birth rates, rising divorce rates, the increasing number of single or unwed mothers, and the growing anti-abortion lobby and signal a growing complexity in the cultural understanding of the role of the mother (Stone 2007, Hays 1996, Chesley 2011). Feminist scholar, Sharon Hays (1996) theorized that the cultural response to the women's movement was the creation of a new cultural narrative about what it meant to be a "good mother". This new narrative, called intensive mothering, was exhausting.

Today's mothers face a dizzying array of conflicting messages about motherhood, married life, and their personal identities outside of their families that today's fathers do 
not share. That is not to say that fathers do not play a vital role in family life, but rather to note the disproportionate pressure experienced by women. This new narrative of intensive mothering magnified this role resulting in a narrative that consolidating parenting duties in ways that result in mothers being "seen as ultimately responsible for the way their children turn out (McMahon 1995, Phoenix and Woollett 1991). Eyer (1996) argues that this narrative works to make mothers into cultural monoliths. That mothers become "little more than architects of the perfect child" (6).

The extent to which a woman internalizes this cultural narrative of being a mother in the age of intensive mothering (Hays 1996) can have a considerable impact on how time-consuming, exhausting, and often precarious the position of mother becomes. It also has important implications for the self-concept that mothers develop. Intensive mothering is not only exhaustive regarding a mother's human resources (i.e. time, patience, emotional support, attention) it is also exhaustive of her economic resources (i.e. finances, wealth). As a result, this ideology of "intensive mothering" and the corresponding identity of 'good mom' have effectively become the white, middle-class standard by which all mothers get measured ${ }^{11}$.

This model of motherhood can be seen as the remnants of the cult of domesticity that told women the way to achieve "true womanhood" was to build a life that centered around their domestic responsibilities as both a mother and a wife; that any failure to meet the social expectations of these roles was an indication of their failure as women

\footnotetext{
${ }^{11}$ https://www.nytimes.com/2018/12/25/upshot/the-relentlessness-of-modernparenting.html?rref=collection\%2Ftimestopic\%2FParenting\&action=click\&contentCollection=timestopics \&region=stream \&module=stream unit\&version=latest\&contentPlacement $=7 \&$ pgtype=collection
} 
(Stone 2007; Edin and Kefalas 2005; Hays 1996 \& 2003; Collins 1994 \& 2005) .

Intensive mothering creates a social expectation that mothers will be ever-present. For mothers who hold competing identities there is a common "rhetoric of shame" that is employed to coerce her back into what has been defined as appropriate motherhood (Stone 2007; Phoenix and Woollett 1991).

Modern mothering is extremely demanding as Hays (1996) notes due to the expectation that the child will both be "the center of familial attention" and "the person who guides the process of "childrearing"" (57). In addition to her dedication to her children a "good mother" will also be suitably-aged, white, western, middle-class, and have healthy children (Lupton and Fenwick 2001; Phoenix and Woollett 1991). This understanding of motherhood has become hegemonic and so pervasive in western culture that women accept and embody it without question (Arendell 1999) although there is research that suggests that this pressure to fit the hegemonic model is not experienced equally by all mothers. For example, mothers in racialized groups and from lower socioeconomic class groups do not experience the social pressure to fulfill the role of intensive mother with the same pressure as white, middle-class mothers (Barnes 2008; Lareau 2003).

However, a growing body of research reveals that many mothers who have adopted this hegemonic understanding of motherhood find it to be isolating, frustrating, exhausting, and demanding in ways that leave them struggling to live fulfilling lives (Crouch and Manderson 1993; Hays 1996; Lupton 2000; Stone 2008). As such, some mothers engage in practices and activities to attempt to fill the identity gap left by the practice of intensive mothering, while others just find themselves falling short. 
Due to the extremely restrictive definition of a "good mother" as defined by the ideology of intensive mothering it is highly likely that many women do not live up to the ideal. Women who return to work or take on college degrees when their children are young, women who devote large amounts of time to extracurricular activities of their own (such as sports), and women who engage in other activities that detract from the time, energy, and resources they should be devoting to their children may not fit the model but they are certainly not subjected to the same degree of social surveillance as drug-using mothers, poor mothers, single mothers, lesbian mothers, or minority mothers (Arendell 2000).

\section{Mothers and drug use}

Drug use for mothers is a behavior, or identity, that requires careful negotiation as they attempt to construct and maintain identities that avoid public condemnation and fulfill the cultural expectations of being a 'good mom'. Management of their drug use alongside with the inherent responsibilities of being a 'good mom' creates a "potentially challenging dual role" (Dodsworth 2012:100) or identity, as the contradictions between the social constructions of the mothering identity and the drug user identity become more apparent in their everyday lives. Mothers who use drugs are subject to the growing pressure to conform the "intensive mothering" ideology despite their social isolation and marginalization in mainstream society. 
A growing body of scholarship focusing on women and drug use has developed over the last thirty years (Ettorre 2004) with much of it constructing women as an at-risk group. This body of research often "reproduces dominant middle-class values with those at risk being categorized as sick or deviant" (Banwell and Bammer 2006: 504, Miller 2001). The addition of research on women and mothers to the literature on drug use has been long overdue it has also been problematic in the way that is has positioned these women as engaging in "riskier" behavior than their male counterparts. This is typically supported by their research due to its focus on drug using women's involvement in "risky behaviors" like sex work, sharing of needles, and having high-risk partners (Breen, Roxburgh, and Degenhardt 2005). This has led feminist scholars to note that the construction of women who use drugs has positioned them as doubly deviant due to their involvement in what has been primarily constructed as a male domain (Broom 1994, Denton 2001).

It isn't only competing identities that women must manage in order to maintain their social acceptance as fulfilling their motherly role. They must also manage behaviors and activities that prior to motherhood yielded little to no social cost or shame. Behaviors accepted as commonplace now take on new social meanings and may now come with social costs that they are unprepared for and unsure how to manage. For example, mothers in the 1980's faced an ever-growing stigma of alcohol use during pregnancy (a practice that was commonplace during their mothers' and grandmothers' pregnancies). A study was published that claimed infants exposed to even minute amounts of alcohol "in utero" were at risk for Fetal Alcohol Syndrome, which was believed to have negative long-term effects on the infant's physical and mental development. 
Numerous studies reported that they could not replicate the study and in fact they had found no signs of any physical or mental impairment in infants born to mothers who consistently consumed 1 to 2 glasses of wine per day (Broom 1994; Emmett 1998). Despite these other studies disproving Fetal Alcohol Syndrome, women who consume even minute amounts of alcohol on rare occasion during their pregnancy continue to face stigma. Because of this stigmatization that threatens to eradicate to the important identity marker of 'good mom' most women in the United States do not openly consume alcohol, in some states there are even laws restricting the alcohol service of women who are pregnant. This social regulation of women's bodies as a result of these disproven claims is also an illustrative example of how the labeling of mothers who use drugs and alcohol as engaging in "riskier" behaviors than their male counterparts has led to a social practice of regulating and restricting the behaviors of women in ways that men will never experience.

In the age of cannabis legalization there are new warnings being issued regarding the drug's use during pregnancy. The Kaiser Health Group published an article proclaiming, "Pediatricians Put it Bluntly: Motherhood and Marijuana don’t Mix" (I really love the play on words here) and citing the American Association of Pediatrics' warning to mothers of the "growing evidence of marijuana's potential harm to children's long-term development". ${ }^{12}$ A claim that echoes back to the warnings given to mothers in the 1980's regarding Fetal Alcohol Syndrome. We now know that moderate alcohol consumption has little to no long-term effects, however, the cultural damage was done.

${ }^{12}$ https://khn.org/news/pediatricians-put-it-bluntly-motherhood-and-marijuana-dont-mix/ 
Something I fear we may already be doing with mothers who consume marijuana while they are pregnant.

These mothers are now marked in two ways. They are considered "bad" or "risky" women as a result of their drug use. At the same time, these women are labeled "bad moms" because they do not maintain sobriety once becoming mothers. This positioning of women as doubly deviant has had a particularly important influence in studies of drug-using mothers despite the use of inconsistent measures and definitions of parenting as noted by Hogan (1998). A significant portion of the research focused on this group has centered on these women's shortcomings and the problems their children encounter by what is conceived of as the result of the mother's drug use.

Drug-using mothers are often constructed as lacking parenting skills, having relationship problems, and having poor upbringings themselves. These qualities are argued to lead these women to be socially isolated, lacking in coping skills, emotionally withdrawn and neglectful of their children, overwhelmingly focused on obtaining drugs, and having difficult partner relationships (Barnard 1999; Barnard and McKegancy 2004; Bays 1990; Denton, O’Malley, Bammer, and Banwell 2002). The work of many scholars on the subject notes that the construction of women, mothers, and their children as "risk groups" often "pit the health, welfare, and, sometimes, the lives of young children against the actions and failures of their mothers who are often seen as 'incubators"' (Banwell and Bammer 2000: 505; Denton 2001). As a result, mothers are often rendered invisible as children take primary positions of concern in these studies.

Several recent studies attempt to work from an alternative perspective to reduce the stigmatizing of drug-using mothers and shift the focus from the harms they may be 
causing their children to more nuanced discussions. Many of these studies center on the argument that a great deal of these perceived harms may be as much related to other social conditions of deprivation and poverty, which is common among drug-using mothers (Emmett 1998; Harrington, Dobowitz, Black, and Binder 1995). Additionally, researchers are also working to show that many of these risks are not solely risks to children of drug-using mothers (Hogan, Myers, and Elswick 2006) and the many ways that drug-using mothers are actively attempting to protect their children from these risks and the effects of their drug use (Richter and Bammer 2000; Jackson 2002).

\section{Belonging}

Allison Pugh's (2009) book, Longing and belonging: parents, children and consumer culture explores the power of contemporary cultural narratives to create emotional meaning that can be used to shape our life choices, our patterns of behavior, and the way we interact with countless social institutions. Her discussion of the meaning making process that is culture becomes vital to my understanding of how the women who participated in this research have come to understand and embody the cultural narratives surrounding motherhood and how that understanding then shapes their understanding and negotiation of conflicting identities they may hold.

"The realm of emotional meaning, wherein neutral objects and events are transformed into things and experiences that matter to people, is the realm of culture. Culture is often defined as a system of meanings, in the words of Sharon Hays 'a social, durable, layered pattern of cognitive and normative standards'...We mix and blend meanings across social realms and experiences, bringing one to another in a daily project of individual 
and collective creativity that nonetheless often serves to reproduce understandings and relationships" (23).

This work and understanding of culture are valuable to the analysis that will follow in the ways that is situates the meaning making process. "Meaning comes from a sort of emotional thinking" that allows how we feel about an experience to "color the ways we perceive them". And culture then can be theorized as "a patterned, collective process by which people attach personal, emotional significance to their world, indeed, as a sort of dynamic, two-way bridge between the social and the psychological” (23).

Belonging is "a sense of membership or acceptance" (Good, Rattan, and Dweck 2012:700) but it also more than that. It is a yearning, a "desire" to feel that kind of acceptance and membership (Pugh 2009). For many of the women who participated in this research, a sense of belonging to a group of other mothers was vital to their own identities as 'good moms'. This feeling of belonging likely has many components, "but at its heart it reflects the feeling that one fits in, belongs to, or is a member of" the community in question. It may also "entail a sense of being valued and accepted by fellow members of the community (Good, Rattan, and Dweck 2012:701).

When considering how this affects motherhood we can see that this desire to belong, to have group acceptance, has had significant impact on the way that women understand and practice motherhood. "To be sure, our trajectories and choices are profoundly shaped by the "organization of human existence," the social institutions, categories, and resources that frame and produce social life" (Pugh 2009:24). A great example of this is the work done by Kathryn Edin and Maria Kefalas in their book, Promises I Can Keep: Why Poor Women Put Motherhood Before Marriage (2005). Their research of poor single-mothers added important nuances to the discussion of why 
women become mothers by examining poor women who are often stigmatized or even sanctioned for their desire to be mothers.

\section{Conclusion}

The focus of this research is on mothers, and as a result, women. This is not to ignore the role that fathers play as parents in contemporary family life. It is acknowledged that fathers are taking on increasing responsibility for a variety of household and parenting duties (Chesley 2011). Despite this shift in cultural practices, "in most cultures mothers are held accountable for the care and emotional development of their children" (Hays 1996). As a result of this cultural expectation placed on mothers, today's fathers are able to focus on their careers and hobbies first and their role as father second, "for the first is directly supporting the latter- sometimes financially, but undoubtedly ideologically" (Eyer 1996).

Beck (1992) argues that modernity has led to a loosening and erasure of traditional norms in society. He refers to this as a "risk society" and argues that individuals in them seek to escape the binding and restrictive social statuses and expectations that accompany them. As social norms about motherhood, marriage, gender,

and sexuality all become less restrictive so do the social expectations associated with each of the individual identities within them. Motherhood has certainly become a restrictive identity in its current form. While it may be true that some women have escaped this binding status by delaying or refusing motherhood, a growing number of women seek to escape the restrictive nature of intensive mothering by shifting the 
cultural narrative surrounding the identity of motherhood. These women are actively engaging in a negotiation of the restrictive expectations placed upon them by what many see as an "outdated" cultural narrative about mothering.

The ideology of intensive mothering makes the identity of motherhood, but more specifically 'good mom', a restrictive identity status that permeates other aspects of her everyday life in ways that are often ignored or overlooked, that is, until she fails to live up to the unspoken expectation. These countless restrictions become the basis for the claim that motherhood is indeed a greedy identity. Women, in general, often find that their behavior is regulated and restricted through social norm regarding gender. Women with children may find that these restrictive gender norms become more heavily enforced and even more restrictive once she takes on the status of mother. If she also carries an identity marker that threatens to complicate, disrupt, or eliminate this status of 'good mom' she must decide if she will attempt to negotiate, modify, or shed the potential. 


\section{CHAPTER THREE:}

\section{RESEARCH THEMES}

\section{Motherhood: The greediest identity?}

The greedy identity of Motherhood

Internalizing the Cultural Narrative

Counternarratives of Motherhood

\section{Practices of Motherhood}

Looking the part

\section{Managing incompatible greedy individual identities}

Individual identity construction

Personal Counternarratives

Holding a competing greedy identity- Marijuana User

The groupwork of collective identity construction

Selective Self-presentation

Online groups as identity generating spaces

Belonging and meaning making practices

Changing cultural meanings one post at a time 


\section{CHAPTER FOUR:}

\section{METHODOLOGY}

The intent of this research is to uncover and elaborate on the individual and collective meaning making processes that shape how mothers reproduce and understand their social identities. In order to ensure that individual, as well as group meanings, were explored a qualitative grounded theory approach (Glassner and Strauss 1967, Charmaz 2001) was taken. My hopes are that this research will ad a layer of understanding to the collective creativity that goes into reconstructing a narrative as powerful as the one that surrounds motherhood.

The data used in this dissertation was collected from 53 semi-structured, in-depth telephone or Skype interviews with women who self-identified as mothers who were marijuana users. These interviews are paired with several months of personal observations in the closed online groups from which participants were recruited. These observations informed the development of the interview schedule, probe, and follow-up questions to ensure that key areas were covered while also allowing individual meanings to emerge from the narratives. My analysis of the resulting data focused on the "identity work" both individual and collective that is taking place in these online groups. 


\section{Interviews}

Interviews seemed the most appropriate research method to achieve this project's goal of understanding how mothers who also engage in marijuana use negotiate the difficult terrain of these competing identities, as well as, marijuana's legal status and their own class status. A myriad of social statuses and meanings impact the ways they choose to negotiate their competing greedy identities. "Interviewing gives us access to the observations of others. Through interviewing we can learn about places we have not been and could not go and about settings in which we have not lived...Interviewing can inform us about the nature of social life". When done correctly interviews can give researchers insight into "people's interior experiences. We can learn what people perceived and how they interpreted their perceptions... We can also, by interviewing, learn about settings that would be otherwise closed to us: foreign societies, exclusive organizations, and the private lives of couples and families." (Weiss 1994:1). Interviews can be vital in understanding identity because they give participants an outlet to define and shape the meaning being attached to their selves.

Interviews allow participants to be active writers in their biographies. Likewise, they give the interviewer an opportunity to "establish an understanding with the respondents that is their full story we want and not simply answers to standardized questions" (Weiss 1994: 3). Women make unique research subjects in qualitative interview studies because of the systemic oppression that exists for all women within a patriarchy, to some extent, often works to limit their avenues to tell their full stories. Despite this, Feminists have paid exhaustive attention to the word woman (Lorber 1193, 
1998; Butler 1990), both how to resist the essentialist definition that implies sameness and the feminist perspective that urges an examination of the many shared experiences of women as well. For example, most western women are likely to grow up with some degree of negative meaning attached to their bodies; to fear rape; to earn less than equally educated men in the labor force, to do more than half of the domestic labor in their homes; and to rely on the services of men in positions of power (Rogers 1998). This interview research is essentially feminist in its efforts to "expose and redress women's invisibility as social actors" (Reinharz and Chase, 2001: 223). "For women there have been thousands of years of silencing. The speech-act itself is a rebellion act against stifling social norms which call for women's silence" making the interview a site of liberatory knowledge production.

For this research I utilized what can be described as "grounded theory interviewing". "A grounded theory interview can be viewed as an unfolding story...conversational in style but not casual in meaning" (Charmaz 2001: 690). In order to do so I employed grounded theory when creating the interview schedule to be used. Initial open-ended questions were used to encourage participants to share their full stories. For example, "Tell me about an average day for you". Allowing the participant to include a level of detail that they are comfortable with, while also allowing the researcher to identify key or important themes to engage with follow-up questions. This again is consistent with the goal of adding women's experiences to the academic discussion while still allowing women to be the authors of their own biographies. 


\section{Recruitment}

The mothers for my study were collected primarily through calls for volunteers in two closed online groups that are organized around the identity of mother. Before continuing it is important to explain what a private online moms' group is, why an individual might join one, and how discussion and interaction takes place in them. In these closed groups discussions and interactions occur in message board form, meaning most of the posts are followed-up with a response from another member, and that response is then followed by a response to that response, and then another, so that the thread goes on and on (Moore 1995). According to Barnes (2001), this type of exchange facilitates the sharing of information while also blurring the distinctions between past, present, and future. The post that result feel more like an ongoing dialogue than an asynchronous communication (Collett 2005).

These are not public groups, meaning only those who are members can see and participate in them, so snowballing from the initial contacts was encouraged. As a result, the sample that was collected must be considered illustrative, rather than representative. This distinction allows for a focus on the discursive boundaries shaping their identities rather than drawing conclusions about the factors that may be influencing their experience of "self".

The setting chosen is of particular interest, because private social media groups provide people with online cultural spaces where they are less likely to experience negative social attention regarding particular identity markers. Unlike public social media spaces that are often cultivated projections of a person's life to portray them in a 
particularly positive light, these closed groups allow women to expose their flaws, confess their failures without judgement, and to challenge the cultural narrative surrounding motherhood. Much like the consciousness raising groups of the 1960's, private social media spaces can provide moms with a space to share their lived experiences, to admit their shortcomings, to ask questions they would not ask in public spaces, to discuss their real lives with other women, and to actively resist the cultural narrative surrounding motherhood. These groups allow women to actively engage in both the collective and the individual identity work that is necessary to resist and rewrite the cultural narrative of motherhood. Because of their online nature they are also accessible to these women from anywhere and at any time of day. Unlike traditional physical support groups members of online groups can log on from anywhere provide they have Facebook access.

A key reason for selection of these two particular online mom groups was that as private groups women felt comfortable to be quite open about many of their "stigmatizing identities" (i.e. their marriage problems, struggles with their children's behavior, their marijuana use...) while posting inside the groups. This openness gave me access to a group of mothers who admittedly carry both the identity of mother and marijuana user.

These online groups are exclusive in many ways that also contributed to my decision to gather my participants from them. First, they are all female spaces. No men are allowed membership in either of these online groups. I believe this gender exclusivity is important for a variety of reasons, but most important is that this exclusivity allows collective identity to occur unabated by outgroup, or male, voices. I also find this female 
exclusive population to be relevant and important to this research because past research has positioned drug cultures as male-dominated. Recently, the construction of drug-use as an activity disproportionately attached to male identities has been challenged and women's experiences with drug use have begun to add a understanding of contemporary drug use. For the above reasons, these closed online spaces become interesting research sites specifically because women are not competing with male expectations of their identities.

Second, these closed online groups were only open to women who were mothers; to be a member of the group you must be pregnant or have at least one child living at home. This allows examination of the intensity of the power the cultural narrative surrounding motherhood and the socially expected practices of motherhood that lead women to experiences themselves as refracted (Bailey 1999) and to see how negotiating and constructing the identity of 'good mom' becomes a group activity and not solely the individual work of the mothers interviewed.

Third, they are by invitation only. Every member of the group was invited to join by another member of the group. I believe this creates an openness for marijuana using moms that may be hard to find in other social spaces as there is a sense of belonging that is created through this type of membership, each mother is able to position themselves as the friend of a friend and perhaps shield themselves from some level of social sanctioning as a result.

Lastly, both groups provided the assumption that member mothers in these groups would be less likely to strictly adhere to the culturally accepted behaviors for 'good moms' and therefore more likely to contain moms who would use marijuana. The groups 
selected were ones that overtly stated or implied in their descriptions that they rejected or at least challenged many of the practices associated with intensive mothering. The groups often described themselves as being for working moms, single moms, moms who attend college, moms who drink and swear, and "misfit" moms.

\section{Data Collection}

Interviews lasted, on average, one hour and were conducted via Skype or telephone and digitally audio recorded. Follow-up interviews lasted, on average, 20 minutes and were generally conducted in the same format as the initial interview. The exceptions were five follow-up interviews that were conducted via email. All of the interviews and transcriptions were completed by me.

As a mother and member of both closed online groups, I was able to use my own experiences as a mother to build rapport with the mothers in my study during my interactions with them on various threads. Despite this superficial rapport, I am unsure of what my respondents thought about me as a researcher, what they believe my intentions to be, or how they edited and organized their responses given their limited online interactions with me. I did feel that most of the respondents were open and forthcoming about their experiences.

A small number of respondents (7) explicitly expressed gratitude for being given the opportunity to share their thoughts and experiences and that someone was taking a genuine interest in their everyday lives. The grounded theory interviews "may yield more than data for a study. Research participants may find the experience of being interviewed 
to be cathartic, and thus the interviews may become significant events for them.

Furthermore, participants may gain new views of themselves or their situations. Many participants gain insights into their actions, their situations, and the events that shape them" (Charmaz 2001: 691).

The interviews for this research were focused on these women's understandings of motherhood and what it means to be a 'good mom', their perceptions of how their marijuana use affects those understandings, and how they negotiate the contradictions that exist between their identity as a mother and their identity as a marijuana user. The interviews were loosely structured and utilized a conversation style to uncover the narratives these women use and to provide a way to reflect on the common practical concerns and practices these mothers employ to situate themselves as both 'good moms' and marijuana users. All the interviews began with an open and broad question such as "Tell me about a typical day in your life" and encouraged respondents to discuss what was important to them rather than allowing my questions to dictate the relevant parts of her day.

Respondents were asked about their attitudes towards, use of, and experiences with marijuana and how motherhood has affected those attitudes. Interviews were centered on their everyday practices and experiences in order to better understand how these women manage the gaps between their understandings of the culturally constructed "good mother" and their real experiences with mothering. To stay true to my commitment to feminist epistemology, I aimed to uncover "truths that illuminate varied experiences rather than insist on one reality" (DeVault 1999: 3; Smith 2005). Through the process of the interview and through writing about the lives of these women I have been better able 
to understand the intricacies of their everyday and the social processes, structures, and meanings underlying their identities as mothers. All the names of respondents used in this dissertation are pseudonyms.

My research is influenced by case study methods, in that it is an in-depth study of a particular group chosen because it is uniquely situated in the social context to reflect, exaggerate, or even counter "social conflicts that our theories suggest are experienced in the wider society" (Williams 1991: 225). "The strength of a case study lies in its depth" (Elliott et al. 2015: 355) which is why focusing on respondents who experience similar conditions and focusing on a narrow group allows researchers, like myself, to uncover and unpack the complexities of these individual identities. It is precisely for this reason that I took care to select a sample that had both illustrative and theoretical value. I believe this sample allows us to formulate understandings of these women's individual identity work and the group work that is so important to changing cultural understandings and has much broader implications for mothers outside of the sample population.

This study is different from previous research on the topic of mothers who use drugs because its focus is not on socioeconomically disadvantaged or otherwise stigmatized mothers who engage in drug use but rather on a group of mothers who on one hand, fit into the hegemonic norm in many ways. While on the other hand, they also engage in drug use. By focusing on mothers who are not otherwise stigmatized we are able to develop a better understanding of the power of the stigma associated with marijuana use on motherhood. 


\section{'Good moms'}

All fifty-three of the women interviewed had at least one biological child, several of the women were also step-mothers to at least one additional child. The ages of the children ranged from infants to teenagers. Four of the mothers reported currently being pregnant with a child. The majority of the mothers were women who responded to a recruitment post. Five mothers were recruited through the snowball method. Of the fiftythree women interviewed for this study ten of them lived in states where marijuana use has not been legalized, either for medical or recreational purposes. However, twentythree of them lived in states where marijuana has been legalized for recreational purposes.

The majority of the mothers included in my study fit the hegemonic model of a 'good mom'. They are mostly middle-class, with forty-one out of the fifty-three women reporting annual household incomes greater than $\$ 50 \mathrm{k}$ annually. The median annual household income in the United States, according to the 2016 Census, is $\$ 51,600$ meaning most of the mothers who participated in the study live at or above the median annual household income level for the United States. Two of the mothers reported incomes greater than $\$ 200 \mathrm{k}$ and two of the mothers reported living below the U.S. poverty threshold for their family size ${ }^{13}$. They were well-educated with thirty-four of the

\footnotetext{
${ }^{13}$ Respondent reported family of four ( 2 adults and 2 children) with income less than $\$ 24,858$. Respondent reported family of four ( 1 adult and 3 children) with income less than $\$ 24,944$. These numbers correspond to federal poverty thresholds. https://www.prb.org/insight/u-s-poverty-thresholdsand-poverty-guidelines-whats-the-difference/
} 
mothers reportedly having at least a bachelor's degree and six reported having either a master's degree or PhD's in their field.

The women in my sample were overwhelmingly heterosexual, with forty-nine of the fifty-three mothers reporting to be heterosexual and thirty-five of the women reporting having current husbands or male live-in partners. Respondents were between the ages of 24 and 44, all within of culturally accepted age for motherhood (mid-twenties to late thirties when giving birth).

The sample was primarily White; forty-seven out of the fifty-three mothers interviewed. Three respondents reported being Hispanic, two reported being AsianAmerican, and two reported being African-American. Although there is some variation in socioeconomic status, marital status, sexuality, age, age of their children, and the racial make-up of the families of the women interviewed the sample is still one that represents the idealized cultural standards of motherhood for the most part.

For some the homogenous nature of my sample may appear problematic, I believe the homogeneity becomes its greatest strength. It allows us to examine the complexities that exist in these women's lives despite their outwardly homogenous make-up. By doing that we are able to see how these seemingly similar women experience motherhood and their own marijuana use in extraordinarily different ways. This is an important contribution to the discussion surrounding motherhood and drug use and vital to our understanding of the ways "the impact of stigma is never homogenous" (Wiegers and Chunn 2015:43) and that each woman will experience it in varying degrees and ways in her own daily life. In addition to the varying degrees mothers will experience stigma there has also been research that shows how mothers in racialized groups and from lower 
socioeconomic class groups do not experience the social pressure to fulfill the role of intensive mother with the same pressure as white, middle-class mothers (Barnes 2008; Lareau 2003).

While this sample may not allow for a deep understanding of how women in social positions outside of the hegemonic model of what constitutes a 'good mom' negotiate the multiple problematic identities they carry, it does allow us to understand the power of the identity marijuana user more fully. Although most of the women in the sample fit the hegemonic model of 'good mom' making their obvious "spoiled identity" that of marijuana user, they also belong to online groups that appear to challenge that hegemonic model in a variety of ways. For example, two of the online groups specifically list in their group description their ideological rejection of the hegemonic understanding of 'good mom' by stating: "Being a mother is hard, we aren't here to make it harder. No judgement" and "Don't be a C***, Kara"14.

It is my contention that this initial framing of the online space as one that is open to the rejection of the cultural narrative of motherhood, while also hosting a relatively homogenous population that seems to fulfill the cultural narrative of 'good mom', is an ideal setting to examine the contradictions between the cultural narrative surrounding motherhood and the lived experiences of actual mothers. I believe the homogeneity of the sample allows this piece of research to uncover the power of this particular "spoiled identity". It highlights the greedy nature of the identity "marijuana user" to create bad moms in ways that may not be as apparent in other research that situates these 'good

\footnotetext{
${ }^{14}$ Closed and secret groups on fb generally have an invite only or approval only membership policy. Part of that membership is often agreeing to set of rules that outline the boundaries of interaction in that particular online space. This is true for all of the fb groups used for the purposes of this research.
} 
moms' alongside mothers who carry other problematic identity markers; such as single, poor, addicted, Black, or even sex workers (Dodsworth 2012; Banwell and Bammer 2006; Elliott, Powell, and Brenton 2015; Wiegers and Chunn 2015).

\section{Materials}

Interview participants were asked to complete a brief demographic survey prior to their interview. The survey was designed to provide background data that could be used to make generalizations of interview results. Both the demographic survey and the interview prompts used for this research are available in the materials appendix at the end of this work.

The interview schedule is consistent with both the goals and the practices of qualitative grounded theory interviews. Questions were semi-structured and open ended to allow participants to shape the important themes based on their own personal lived experiences. The interview schedule included questions on the nature of their marijuana use, if they felt that their use was socially acceptable, and what practices, if any, they employed to reduce social stigma that is associated with motherhood and drug use. Questions specifically relating to how these women defined 'good mom' and how similar or different their understanding of that title and the cultural narrative surrounding it were also included. Emphasis is placed on the ways these women understand themselves to be 'good moms' despite their identities as marijuana users. This problematic identity threatens to disrupt or negate their prized identity of 'good mom'. Participants were asked 
if/how they negotiate these two competing and greedy identity markers and their motivations for doing so. 


\section{CHAPTER FIVE:}

\section{"I'm a good mom": The greediest identity}

"I think I am a good mom. I am always amazed by the connection I have with my son and the feeling that I understand him, and I know what he needs, even though he's largely unable to communicate it to me at this point in his life. I watch him carefully all the time, while simultaneously working very hard to resist the urge to jump in any time he falls or encounters a problem because I want him to learn how to navigate life's challenges independently. It's hard for me, but I believe it's good for him in the long run. I make mistakes, but I always make sure that he knows he is loved. So, I do everything I possibly can to make sure he has everything." - Rachel, 34

\section{The greedy identity of motherhood}

In his book, Peacocks, Chameleons, Centaurs: Gay Suburbia and the Grammar of

Social Identity, Dr. Wayne Brekhus uses motherhood as an example of a greedy identity.

He argues that it can be seen as a greedy identity that many women hold as it "requires

exclusive and undivided identity work" (2003:45). He is careful to point out the important reality that this identity may not be experienced in the same way for all

mothers. He states:

"Mothers of infants, for instance, are practically forced to become mother lifestylers, as motherhood at that stage is a greedy identity that allows little room to commute to other selves or to integrate many other attributes. Whereas mothers of older children can lifestyle, commute, or integrate, the high-intensity, high-duration demands of a newborn infant and the high demands U.S. society places on mothers of infants to be full-time caregivers require the type of undivided high-density commitment that only a lifestyler can provide. Although we may value integration in other realms, most individuals in the United States want mothers of newborns to be fully committed to their mother identity, even at the expense of all other identities." (143). 
The interview participants in this research confirmed the claims made in Dr. Brekhus' work. During the interviews with the participants that were pregnant or currently had infant or toddler aged children I found that they demonstrated almost entirely what Brekhus outlined as characteristics of lifestylers (33). Lifestylers, which he equates to peacocks ${ }^{15}$, choose to live their social identity at maximum intensity for maximum duration. It was not surprising then that new mothers who internalize the narrative of intensive mothering became lifestylers of the mother identity; experiencing it at maximum intensity for maximum duration.

In this sense, these mothers "exhibit an identity in its seemingly most pure and unadulterated form. They are the cultural vanguard of identity politics, and it is their aesthetics, values, and tastes that publicly define and trickle down to other members of the identity group" (Brekhus 2003:46). The "lifestyler" mom "lives and experiences (her momness) as an omni-relevant social attribute" meaning that for her it becomes a "master status socially, politically, and phenomenologically" she "most clearly illustrates the consequences of social markedness and captures the concept of master status in its most ideal form" (47).

Yesenia, a 30-year-old mother of three children under the age of six provided a clear example of this. When asked to describe a 'good mom' in her interview, she gave an answer that was clearly shaped by an internalization of the intensive mothering

\footnotetext{
${ }^{15}$ Brekhus uses lifestylers (peacocks), commuters (chameleons), and integrators (centaurs) to discuss the way that those who hold gay identities while occupying suburban life embody and display that identity. He found that lifestylers lived these identities visibly at all times, while commuters moved in and out of various identities. Commuters would live an identity at a high intensity for a short duration. Finally, integrators were those who lived multiple identities simultaneously. They found ways to live lower intensity identities and negotiated multiple identities within a given social context.
} 
ideology. "A good mom is someone who is attentive of her children and their needs. A good mom is involved with their schooling and decision making. A good mom makes sure her children are bathed, clothes and fed and ensure their child know kindness. A good mom is emotionally and physically available for her children, and if she can't be, makes moves to ensure she is striving to be".

Other mothers with infants and small children had similar sentiments about what it meant to be a 'good mom'. Ellen is a 39-year-old mother of a 1-year-old son who stresses many of the ideologies of intensive mothering in her description. She stresses her desire to be a 'good mom' because she delayed motherhood and now feels that her son will be an only child as a result. "I waited for so long to have a baby. It's the most incredible thing. He will be my only, so I am so thankful for every experience. Even the tough things I know will soon pass. It is important to me that others see me as thinking of my child first. I try hard to feed his mind body and spirit and allow him to explore and learn but be safe."

Dawn, a 38-year-old mother of two girls under 2-years-old who works full-time as a college professor, was quick to point out that she was a mom no matter where she was in relation to her children and no matter what "role" in her life she was actively doing.

"I am constantly doing or thinking about mom stuff. Even when I am not at home with them. Like, on my way to my classes I find myself wondering if they have napped, or eaten, or if they are happy or sad. I worry about things like if they are developing at 'the right pace' and if I am doing everything I can to make sure they are getting everything they need to grow. There are days that being a mom can get in the way of other parts of my life. It is hard to prioritize anything over them. Even when life demands that I do because they are always with me. I think about them more than anything else. They are the only part of my life I never put down, if that makes sense." 
It is easy to see why motherhood is theorized as a greedy identity as it "washes over other aspects" of a woman's life and pervades all of her social networks (Brekhus 2003:45). Under these social expectations, motherhood becomes an all-encompassing identity that leaves women struggling to negotiate all the other parts of their identity in a tenuous tightrope walk through their everyday lives.

Researchers have found that women of all racial groups and class backgrounds overwhelmingly hold to intensive mothering as the ideal for motherhood. Even when there are structural barriers to their ability to achieve it, they internalize this as the model of motherhood that they believe must work towards. The extent to which a woman internalizes this cultural narrative of being a mother in the age of intensive mothering (Hays 1996) can have a considerable impact on how time-consuming, exhausting, and often precarious the position of mother becomes.

This modern approach to mothering requires that women are self-consciously committed to child rearing ${ }^{16}$. It is a "greedy identity" that expects women to be childcentered, expert-guided, emotionally absorbed, labor-intensive, and financially dedicated to their children in a way that erases other facets of themselves that existed prior to becoming mothers (Hays 1889; Edin and Kefalas 2005; Stone 2007).

Women with infants are not the only mothers who may find it difficult to escape the lifestyler identity of mother. Joni, a 36-year-old mother of 10-year-old fraternal twins who are "severely autistic" and require constant attention, pointed out the greedy nature

\footnotetext{
${ }^{16}$ New York Times article published 12/25/2018. "The relentlessness of modern parenting". https://www.nytimes.com/2018/12/25/upshot/the-relentlessness-of-modernparenting.html?rref=collection\%2Ftimestopic\%2FParenting\&action=click\&contentCollection=timestopics \&region=stream\&module=stream unit\&version=latest\&contentPlacement=7\&pgtype=collection
} 
of her 'good mom' identity during her interview. When asked to describe an average day in her life she responded,

"I am a mom. Every second of every day. I am a mom. And most days I am not sure that is enough. I wake up and if I am lucky I get a few minutes to drink my coffee (sighs) before I have to just be mom. I spend my days taking care of other people. My kids are my responsibility and I take it serious. I work hard every day to make sure that they have everything they need and that I am doing extra stuff so that they have a better life. I used to have a life of my own. Now they are it. My kids really are my whole life. Their mom that is who I am."

That is not to say that mothers whose children do not have developmental/physical/or learning disabilities voiced experiences that were dramatically different from Joni's in many regards. The vast majority of all the mothers interviewed declared that they spent the vast majority of their time caring for and mothering their children. There were variations in the amount of time doing physical mothering occupied in their days, with mothers with small and developmentally/physically disabled require the most physical mothering. On the other hand, mothers of teens reported spending large amounts of emotional and intellectual mothering.

Sarah, the 41-year-old mother of two high school aged children expressed this consolidation of labor into the emotional realm in her interview. "They do a lot for themselves these days. She is a Senior with her own job and her own car. He is a sophomore now, and he is always gone with basketball. So mostly I just worry. That is how I mother them. I worry. We text. It is nothing like when they were little, and I had so much to do".

That is not to say that only the mothers of teens felt they did an overwhelming amount of emotional mothering. Leigh, a 37-year-old stay-at-home mother of two preschool aged children also discussed the time and emotional commitment she makes to 
her children when discussing a typical day. "My children are my world. They are the first ting I think of when I wake. 'What should they wear today? What am I going to feed them? Will we have time to do a fun activity? Are they going to have a good day?' I put a lot of time and energy into thinking about how their day went and making it as good as I can."

In this context, it is easy to see how mothers become cultural monoliths. Flattened narratives that leave little to no room for a more nuanced understanding of the multiple, complimentary, and conflicting identities that they may hold. While being a mother is only one of the multiple statuses that a woman may occupy, albeit often an extremely important part of their status set, in many cases it becomes their master status that eclipses and permeates all other statuses they hold to some degree (Goffman 1964). Dodsworth (2012) points out that,

"despite recent discourses that identify the existence of a diversity in maternal identities and a growing recognition that women with children are not only mothers but have other relationships, employment and identities notions of the 'good mother' continue to be linked to social constructions of the 'ideal mother' who either does not work outside of the home or, if they do, prioritizes their children's welfare” (p.100).

The stay-at-home mom, regardless the age of her children, is another example of the lifestyler mom we can identify because of the way the identity becomes high in both intensity and duration. Many of the stay-at-home moms in the study referred to being a mom as her job. Much like occupational identity becoming a crucial part of a person's individual identity when they engage in the labor market, so does mothering for those women who do not engage the wage labor market. 


\section{Internalizing the Cultural Narrative}

During the interviews there were four main characteristics that were routinely associated with being 'good moms' and that eventually became the scaffolding for this analysis . They were being selfless or self-sacrificing, being "there" for your children, demonstrating love and acceptance, and finally showing support or helping. It was fairly predictable that these attributes would be important to the mothers' understandings given the power of the cultural narrative created by the ideology of intensive mothering.

For decades media representations of mothers and experts on families in the United States have actively promoted the ideal of a 'good mother' as a mother who devotes unlimited amounts of time, love, patience, energy, and resources to child-rearing, even if it means sacrificing her personal needs, wants, and aspirations (Warrior 1986; Boulton 1983; Brown, Small, and Lumley 1997; Lupton and Fenwick 2001; Stone 2008; Edin and Kefalas 2005; Hays 1996). Over the course of the interviews for this research project it became quite clear that many of the mothers participating had internalized the cultural narrative of intensive mothering, the societal expectations that mothers would/should embody all of these qualities in their practice of motherhood. Some of the mothers made this more central to the narrative they constructed about what it meant to them to be 'good moms'. 


\section{"Self-sacrificing moms are great moms!"}

Many of the mothers called upon this notion of mothers being selfless. There was an acceptance that mothers would sacrifice for their children. Mothers were expected to sacrifice careers, educations, hobbies, recreation, and countless other practices and identities in the name of their children. At times the identity of selfless mom became a badge of honor that mothers wore proudly in their exchanges in the group. Single-moms frequently posted about the things they wanted but would not buy because their child wanted something instead; a way of undoing the damage done to her identity of 'good mom' by the fact that she was single, or poor. The attribute of being self-sacrificing carried a weight that enabled it with the power to restore a part of their identity.

Traci, a 41-year-old mother of a 4-year-old son, demonstrated one way that this cultural narrative of intensive mothering contributed to her understanding of motherhood. When asked to describe a 'good mom' she responded, "I think a good mom is someone who cares for her child/children and does everything she can for them. She would be selfsacrificing and dedicated to doing the best they can". Traci was certainly not alone in the importance she placed on being "self-sacrificing". A 37-year-old mother of three teenagers, named Amanda, also supported Traci's sentiment with her description of a 'good mom'. For Amanda a 'good mom' was, “any mom that puts their children before

themselves". She followed up her description with "self-sacrificing moms are great moms".

Other mothers were not as direct in their descriptions, but it was clear that they expected mothers to be "selfless" or self-sacrificing in their practice of motherhood. For 
example, Ellen, a 39-year-old mother of a 2-year-old, stressed in her description of a 'good mom' that she would always be "thinking of her children first and putting their needs before her own”. Mal, a 28-year-old mother of one small child agreed that good moms "should" have all of the qualities mentioned above. In addition to these she believed that 'good moms' are "honest, hardworking" and have a willingness to "do anything for their child".

For a few of the mother's in this study their internalization of the cultural narrative was not as apparent in their definition of a 'good mom' but rather in their discussions of who they looked up to as role models of motherhood and why those individuals were significant in shaping their ideas about being a 'good mom'. For example, a 43-year-old mother of a teen daughter named Sarah discussed how she learned this model of motherhood from watching her own mother. "When I think of a 'good mom' I think of my mom. She tried hard to be there for us and be an active participant in our lives, she was and is present when we talk to her, she put us first in her plans and always included us in her decisions".

Another mother named Sarah, this one 40-years-old and the mother of two teenage children in a blended family looked to her grandmother for what it meant to be a 'good mom'. "My grandmother was a military mom who traveled and made sure all her kids had what they needed. Put her kids before any of her needs or wants". These women have clearly internalized the cultural narrative of intensive motherhood in their understandings of what it meant to be a 'good mom'. It is worth noting that these women all reported annual incomes of $\$ 100 \mathrm{k}$ or higher, meaning that they were all squarely middle-class. 
Another way this idea of being selfless manifested itself during the interviews was the ways that mothers expressed "mom guilt" for engaging in behaviors that they or others had deemed as too selfish, or self -indulgent, for mothers. The work of McGannon, McMahon, and Gonsalves illustrates this by examining how physically active mothers use recreational sport as an avenue for social and cultural barriers to be renegotiated in ways that promote well-being and sport participation after children (2018). They argue that the "cultural norms of good mother and care giving ideals" work to "subordinate women's physical activity pursuits" (41). It is my contention that the same could be said about any identity that is organized around activities that might be perceived as detracting from a woman's ability to fulfill the social expectations of motherhood. Cary, a Postdoctoral Researcher, discussed how others in her life had attempted to make her feel guilty about pursuing a graduate degree as a single-mother of 3. "Society says that moms should be selfless and give up things for their kids. I think the opposite is true. That would make me miserable and miserable Cary is not good for anyone. How can I be a good mother if I am miserable every day? And poor?".

\section{“Good moms are present and sober, right?"}

A second recurring theme that became apparent during the course of this research was the importance that mothers placed on time spent with their children or just being "there" for them. This notion of being "there" was more than physical proximity, although many of the mothers did stress physical presence. In this case, being "there" for their kids implied a more nuanced meaning. Britney, a working-class, single-mother of 
two boys aged 6 and 9 stressed the importance of being "there" for her boys in her description of what a 'good mom' was. She stated, "a good mom is one who is attentive to the needs of their kids, who puts their safety and emotional well-being above all," a sentiment that was supported by other mothers' interviews as well.

The idea of being present and sober become tangled in a web of collective meaning about the demands and responsibilities assumed in the role of 'good mom' and the understood effects of a variety of intoxicating or altering substances, the main focus being placed on marijuana (probably the result of the nature of the interviews) although many of the mothers also mentioned alcohol consumption. This became more apparent when the interviews shifted to the practices of these women when they engaged in marijuana use. As a result, the idea of 'good moms' being sober moms will be discussed in more detail in the following chapter.

Laci, another working-class mother of two children who is the sole provider for her family also stressed the importance of being “there". For Laci, a 'good mom' was "Someone who is there for their children, supports them and helps them make decisions. Provides their needs and some wants". Many of the mothers who were interviewed relied on examples of women they knew to illustrate what they meant when they said a 'good mom'. Estelle, a 39-year-old, divorced mother of three children demonstrated this in her interview, "I have several friends I consider to be good moms. They do what they need to in order to provide financial stability and necessities for their families. They spend quality time with their kids. They are emotionally supportive and take a vested interest in their kid's activities and friends. They have found a healthy balance between mom and self. ". 
Sarah, who drew on her experiences with her grandmother to describe a 'good mom' also drew on examples of women from her own life to discuss this important act of mothering referred to as being "there" for their kids. "My mom tried really hard to be there for us. And to be an active participant in our lives, she was and is present when we talk to here, she put us first in all her plans and always included us in her decisions. We always felt important." Mothers of all class backgrounds included this idea of being “there" in their narratives about 'good moms' but it was evident that my mothers who reported making $\$ 45,000$ or less annually were more likely to stress this attribute as one that made an individual a 'good mom'.

\section{"She loves and accepts her children"}

Mothers of all social classes, family form, racial identity, and sexuality had clearly internalized the cultural narrative that mothers are supposed to be loving and accepting of their children. Katey, a 28-year-old single-mother of a 7-year-old boy pointed to these qualities as being the most important in her definition of a 'good mom'. She stated, "A good mom is someone who loves their child and obviously wants to help them grow”. For Katey, love becomes key to good parenting. Much like the idea of being "there" for your kids, it appeared that mothers with lower socioeconomic statuses placed more emphasis on these types of qualities in parenting. These tend to be things that mothers can give freely, by defining a 'good mom' by her abilities to give of things like her time, energy, and love these moms have already begun the important work of shifting the narrative. 
Kim, a 40-year-old working-class, single-mother of three children under the age of 4 also stressed this as being key to her understanding of what makes someone a good mom as well. For Kim, “a 'good mom' works, loves her kids, spends one on one time with each of them, creative, thoughtful and patient". Again, all qualities that come with very little financial obligations for these working-class moms to fulfill.

\section{"Always there to help and support"}

Mothers, much like wives, are defined as "domestic help" in a sense. So, it should come as no surprise that the women in my interviews often called upon a woman's ability to help and support her children as a characteristic that may warrant them the title 'good mom'. Laci also discussed the importance of supporting her kids. For her, it was these specific qualities of Love and support that were essential to earning the title.

As the children of the women aged, the emphasis on support rather than presence grew, as many of the mothers with younger children had emphasized in their interviews. Amanda, a 37-year-old stay-at-home mother of a 16-year-old son and 13-year-old twin girls; Cary, a 44-year-old mother of 14-year-old twin boys and a 12-year-old daughter, who works as a Postdoctoral Researcher; and Sarah, the 40-year-old mother of an 18year-old daughter and a 17-year-old son, who works as a Personal Banker are all great examples of this. Each of these women stressed this quality of support in mothering and either used it in their description of, or their reasoning for why they believed they were a 'good mom'. 
Each mother had her own way of defining support. For each it was clear that this was the most important characteristic to them when mothering a teenager. Amanda's response was about supporting her kids' development by living an "honest life" with them.

'A 'good mom' in societies eyes is one who upholds an ideal of perfection. It's hard to realize and admit that a "perfect mom" is not necessarily a 'good mom'. A good mom struggles and admits their faults, a good mom asks for help, a good mom allows their children to see the struggles and sacrifice daily and communicates those struggles and sacrifices so the children learn that it's ok to stumble and fall, but to keep moving forward no matter what. This is how I support my kids. I show them being an adult is difficult and that it is okay to struggle. Because they are gonna struggle."

Cary also discussed the importance of letting her older children see her as a person with flaws and struggles. Both women stressed the importance of allowing their children to see them as flawed so that when their child fails or struggles they will know that she did also, and that she will continue to love her children unconditionally.

"I think of a good mom communicates with her children as if they will someday need to do this whole life thing on their own. I think a good mom does the best she can at any given time and cuts herself some slack. A good mom models for her children what it looks like to be true to yourself and be a bit selfish when making choices. A good mom recognizes that before she can be a mom, she must be herself. I think my children first see me being a good woman. They see me chasing my dreams, having failures and dealing with it, struggling and hustling to get what I want for them and myself in this world. I think this is important so they can accept their own struggles too."

Sarah's answer made it obvious that she had internalized the cultural narrative of intensive motherhood a bit more than Cary and Amanda and it was evident when asked if 
she believed she was a 'good mom'. 'Yes, I am a good mom. I provide everything that my children need. Love, support and care for them. I am a married mom, but I parent like a single-mother. I never miss any extracurricular activities and try to put them before $m e$ ". For Sarah, supporting her children meant being present for events that made her children proud. It meant cheering them on, taking pictures, celebrating victories, recovering from defeat, and showing them that, "what is important to them is important," to her.

\section{Counternarratives of motherhood}

A significant portion of the mothers in the sample voiced a lower level of internalization of the narrative of intensive mothering than many of the mothers discussed in the previous chapter. The narratives of these mothers demonstrated that they held ideologies about motherhood that while often complimentary to the cultural narrative of intensive mothering, also simultaneously rejected some portion of the cultural norms and the practices associated with 'good moms'.

These self-proclaimed 'misfit mamas' were interesting in that for the most part they accepted the ideology of intensive mothering. They believed that mothers should be self-sacrificing or selfless, they believed that love and acceptance were vital, they stressed the importance of 'being there', and being supportive. But there were clear areas of the cultural narrative that they selected to challenge or outright reject with their parenting practices. They were critical of the cultural notion that mothers must be "everpresent" and devote all of their time, energy, and resources to their children. In this sense, 
they challenged the notion that women with children should not work outside of the home or hold any identity that disrupts their ability to live up to the standard established by the intensive mothering ideology.

They had created individual and collective narratives that were used to situate the problematic identities they held; such as elite athlete, working-mom, or marijuana user, as unproblematic to their identities as good mothers (Chesley 2011; Stone 2007;

McGannon et al 2018). These mothers told stories of their lives that demonstrated their ability to be good moms and hold a potentially threatening identity at bay.

Shauna, a 38-year-old mother of two children aged 6 and 3 who holds a demanding career in the federal government, was the first to point this out in the interview process. When I asked her to explain how her ideas about what it means to be a good mom were different from what society tells her a good mom is?

\footnotetext{
"Not substantially different from what we are told, but to a degree. I'd say "society" at large has very different ideas of what a good mom is depending on who you ask - for example, I don't stay home with the kids and I work full-time. I do not attend all PTA meetings, nor all school events. I do not buy arts \& crafts supplies and make little projects with the kids each afternoon. But my husband and I provide for our nuclear family with no help from other extended family, the kids do activities outside school, they are being raised bilingual, and they have plenty of time to come up with play on their own as well. So, I think in that sense I do fit society's picture."
}

Margaret, a 37-year-old mother of two children under the age of 8 who works as a coffee importer in North Carolina, had a similar response when asked about her views "I think my definition is pretty similar to society's definition. I don't include things like fancy trips, designer clothes, or big houses because material things do not make anyone a good parent", when asked if she felt like her definition of what a 'good mom' was and 
society's definition were similar?. Her statement demonstrates her desire to affirm her status as a 'good mom' while also rejecting many of materialistic practices associated with intensive mothering.

Several other mothers in the study also clearly demonstrated how the new narratives they were creating about motherhood challenged traditional notions about mothers who participate in the wage labor market outside of the home. Andrea, a 38year-old mother of a 4-year-old and a Marketing Executive from upstate New York, illustrated this when she emphasized the importance of non-maternal identities to being a 'good mom'. She said that when she thought of someone who was a 'good mom' she thought of her friend Megan, "who sets an amazing example for her two sons by pursuing a career that fulfills her. She's also incredibly patient and answers every single one of their questions with so much care and respect". In this case, I found it interesting that her friend is the mother of boys. I wonder if her behavior would have been met with the same positive response had her friend Megan's children been girls?

Frequently, mothers in the groups are praised for demonstrating behaviors that reinforce traditional gender norms and roles, particularly when they are mothers to young girls. We praise them for being excellent role models to their daughters for demonstrating 'proper' gender roles. Mothers with boys may feel less pressure to conform to many feminine ideals, although some report just the opposite. For mothers of boys the role they are responsible for modeling is one that culturally is thought of as being only secondarily her responsibility. She becomes a model of what her sons' potential future wives should embody. And more so, what good mothers are. In this way she plays a role in shaping the narrative that he will use when he considers who will be the potential mother of his 
children. In both roles her behavior is a model for another significant other's behavior not a template for him specifically.

Several mothers in the study drew upon examples of 'good moms' from significant others in their lives. Typically, these were their own mothers/grandmothers, or other mothers that they had close relationships with. They viewed all of these women as being successful in overcoming a culturally defined or understood "obstacle to" achieving the identity of 'good mom'.

Pamela, a 42-year-old mother of three children under the age of 10 from upstate New York, who had recently separated from her husband was a prime example of this. Pamela is recently a single-mother after 15 years of marriage and when asked who she admired as a 'good mom' she responded,

"I think of many of my friends who are working moms, balancing kids, full time jobs, kids' activities, health issues, some part time businesses, some as single parents, some while caring for parents. I look at them as good moms because they are holding it together and doing the best by their kids with all they've got. I think my ideas about what it means to be a good mom are different from what society says. I think society tells us we have to look and sound and act perfect and I don't agree with that at all. I don't think that's attainable".

During observations in the moms' group that Pamela was a member of, I observed several posts from Pamela that demonstrated she felt she was not living up to her own expectations of what a 'good mom' was since her separation from her husband.

Melissa, a 33-year-old mother of two children under 8 years of age from Vermont who works as an Administrator at a local university, provided a rebuke of the notion that her statuses of professional and mother were in opposition to one another:

"The traditional metrics used to decide if someone was a good mother are becoming outdated. Society has historically said you couldn't possibly be a good mom if you worked out of the house. I don't believe that's true. I 
work, I show up, I take care of my family, and I do it well. Does that mean we eat ham sandwiches for dinner twice a week when I haven't had a chance to prepare something or get to the store? Yes. It does. But that doesn't make me a bad mom!"

Sarah, the mother of a 13-year-old daughter from Washington, provided a similar argument with her answer to the same question only instead of addressing potential stigma faced from being a working-mom she chose to address the stigma she feels she encounters regarding her sexuality. Sarah cohabitates with her FTM partner and often feels that she is judged in circles of mothers she encounters in her every day. For her, being queer is a greedy identity that she must manage.

"I think my beliefs can exist on both sides of the debate. In some sense I am a very traditional mom and meet all of the requirements of what is considered a 'good mom' in our society. However, I also am queer, and give my daughter more independence in deciding who and how she wants to present in this world. Sometimes that makes others uncomfortable."

Melissa and Sarah provided arguments that were both in support of and in opposition to the cultural narrative of normative motherhood during their interviews. This was not a universal narrative, in fact many of the moms voiced clear rejections of the cultural narrative of intensive mothering. When asked if she believed that her ideas about what it meant to be a 'good mom' were different or similar from the stories that society tells women about what it means to be a good mom, a 37-year-old mother of three teenagers, named Amanda responded,

'I think they are different. A 'good mom' in societies eyes is one who upholds an ideal of perfection. It's hard to realize and admit that a 'perfect mom' is not necessarily a 'good mom'. A good mom struggles and admits their faults, a good mom asks for help, a good mom allows their children to see the struggles and sacrifice daily and communicates those struggles and sacrifices, so the children learn that it's ok to stumble and fall, but to keep moving forward no matter what." 
In fact, many of the mothers interviewed shared in this rejection of the notion that to be 'good moms' needed to be perfect. They rejected the relics of the cult of domesticity that claimed that a woman's value could often be seen as being attached to her ability to create a comfortable, safe, and clean home. Mothers in this study actively constructed narratives that de-emphasized many of the domestic responsibilities or expectations that are placed on mothers. These relics from the ideology of the cult of domesticity still haunt the daily lives of mothers like Kristin, a 38-year-old mother of a 1-year-old in Vermont. During her interview she stated that she felt that "society would like a perfect housewife mom. But to me it's about love and quality time together. Who cares if the dishes are done?!". Rachel, another mother from Vermont, echoed this rejection of perfection in her interview,

"I think there are a million ways to be a good mom and I wouldn't presume to know the best one. I also think that we're beginning to see this attitude a lot more in parenting across the board - less judgment and more acknowledgment that as long as mom is showing up day after day and doing her best, she's doing a good job. So, in that way, yes, I think I agree. But the classic notion of mom keeping a spotless home, feeding her kids veggies, and doing clever art projects, blah blah... no. I guess I don't agree with that."

Megan, a 29-year-old mother of two boys aged 4 and 6 who lives in Missouri, stressed the importance of allowing women with children to also be 'human', with her statement she rejects the oppressive often "unattainable" expectations that women with children are faced with.

"I think they are very different. When I think of a good mom I think of someone who is there and understanding. They can be human, have needs, disappointments, problems, and show their children how to work to overcome strife instead of creating some unrealistic image of perfection" 
Her statement also points to the need for a narrative about motherhood that encourages an honest discussion about women's real experiences and how they compare to the cultural expectations placed upon them. Societal expectations concerning motherhood and the cultural norms surrounding intensive mothering are grounded in social constructionism, whereby 'motherhood' is "viewed as the product of individual, social and cultural discourses which interact to create particular meanings concerning mother identity" (McGannon et al 2018:41; McGannon and Schinke 2013).

\section{Conclusion}

Over the course of the interviews there were clear themes that arose about how these women defined and understood themselves to be 'good moms'. After analyzing the transcripts more closely and comparing them to the demographic surveys completed by my participants prior to their interviews I noticed that there was a relationship between the emphasis placed on particular thematic understandings and the social class of the mothers. The four main characteristics associated with 'good moms' in the interviews; being selfless or self-sacrificing, being "there” for your children, demonstrating love and acceptance, and showing support or helping were all apparent to a certain degree in all of the interviews. Something that I found to be interesting was the impact that a mother's socio-economic status had on which of those themes they emphasized in their own 
practices of intensive mothering in comparison to the themes that mothers of lower socioeconomic backgrounds stressed in their interviews.

Mothers of higher socioeconomic standing seemed to stress "being present" and "support" as being the most important traits to being a good mother. For them, being a good mom was about physical and emotional access for their children. These mothers stressed the importance of availability and really getting "to know" their children as people. Mothers who occupied lower socioeconomic statuses, however, seemed to place importance on "Love/Acceptance" above all other traits. For many of these mothers their love for their children was the only trait that they expressed should matter. As long as their children knew they were loved and accepted then it seemed a myriad of other socially questionable practices could be overlooked.

I believe that these differences in understandings of what makes a woman with children into a 'good mom' are influenced by the everyday lives of these women. They are grounded in a materiality that structures their understandings of themselves and the world that they live in. In addition to decades old cultural narratives that attach motherhood and the 'housewife' in ways that become nearly impossible to untangle from one another. Through these conflated understandings we can see how women have become "ideologically redefined as the guardians of a devalued domestic life". (Davis 1992:228).

In this way the 'good mom' becomes as much a mythical reality as notion of women as 'housewives' being the natural order of domestic life (Gilman 1903). The housewife reflects only a partial reality, she is more of a symbol than a reality. An ideal, "an identity in its seemingly most pure and unadulterated form. They are the cultural 
vanguard of identity politics, and it is their aesthetics, values, and tastes that publicly

define and trickle down to other members of the identity group" (Brekhus 2003:46) and as such developing a clearer understanding of what good mothering is and how women practice it will require that we move beyond these mythical understandings and examine the realities of the lives these women live every day. 


\section{CHAPTER SIX:}

\section{Practices of Motherhood}

Motherhood, or at least the identity of 'good mom', can be theorized in a comparable way to how gender has been. Motherhood can also be viewed "as an accomplishment, an achieved property of situated conduct" (West and Zimmerman 1987:126). This chapter examines the "conventionalized gestures" used by women with children to “display” their identities as good moms (130). Following Goffman’s (1976) analogy, motherhood "is a socially scripted dramatization of the culture's idealization of" women with children, "played for an audience that is well schooled in the presentational idiom. To continue the metaphor, there are scheduled performances presented in special locations" (76).

As the ideology of intensive mothering has taken hold in Western society, we have seen the social expectations of how 'good moms' practice, or perform, motherhood have changed. This ideology tells women with children they must be child-centered, emotionally available, financially exhaustive, and constantly engaged in mothering and has complicated their lives in a multitude of ways. The expected practices of women with children can be exhausting. The amount of "expert" knowledge available to parents grows daily, in a variety of forms. Motherhood has been known to trigger voracious reading or consumption of relevant knowledge in mothers; books on pregnancy, books on birth, books on newborns, books on child development, books on nutrition, books on discipline, books on potty training, podcasts etc. This expectation that mothers will seek out expert advice through books and articles may seem insignificant, but it has clearly 
become normative in our society. Rachel, a 27-year-old, middle class, stay-at-home mom of a 3-year-old pointed to her perceived failure for not reading (even if internal),

“Occasionally I feel like a less-than-optimal parent because I don't read parenting books or subscribe to a particular parenting method. These things are pushed a lot in the current parenting culture. But I also think that knowing your own child and doing what works for each individual parent is undervalued (as opposed to an overarching parenting methodology or theory). I failed at breastfeeding, which often makes me feel like a bad mom (even though my kid is 3.5 and would likely be weaned by now)".

Rachel was certainly not alone in her critiques of her own parenting. In fact, some mothers seemed to cast some of the harshest judgement on themselves. The mothers who allowed themselves to have a pass on these minute details also seemed to hold less tightly to the narrative of intensive mothering. Beth, a 34-year-old mother of two children aged 2 and 4, expressed that her beliefs were "similar, although only at the general level (to the cultural narrative of intensive mothering)... I try not to get into the minutiae of parenting advice and instead trust my but and my husband's"

Some of the cultural and social restrictions placed on mothers may seem insignificant to anyone who has never mothered. For those who have, they will be familiar with the ways that motherhood restricts or at least imposes social expectations about a laundry list of social behaviors about where they should live, what they should consume, how they should spend their money, and who they should associate with.

Mothers are expected to live in a community that is "child friendly". There is certainly a social expectation that 'good moms' do the research needed to determine if a community is one that has access to needed resources and is safe for children. She is 
expected to have checked into school districts, parks, crime rates, sex offenders, and proximity to other children close to the ages of her own before making decisions about where she will live with her children. She is expected to provide them with a home that provides them with individual space and privacy, as well as a sense of belonging and togetherness. Even mothers with few resources are expected to find "the best place they can afford" for their children ${ }^{17}$. There are a host of other ways motherhood restricts the behaviors, lives, and identities of women.

Motherhood can also restrict, or at least constrain, the employment choices available to women, a social arena where women have historically been excluded from a significant portion of the labor market. Mothers are often restricted in the hours that are socially acceptable for them to work as the ideology of intensive mothering promotes the idea that mothers should be constantly available to their children. In Pamela Stone's (2007) book, Opting Out: Why women really quit careers and head home, it is argued that demanding, high-powered careers are largely untenable for women with children due to what she argued was a 'choice gap' experienced by women (121). In this case Stone is referring to the difference in the opportunities and experiences of men and women who attempt to occupy a high-powered career and also parent.

Another perhaps more working-class example would be a mother who works a night shift (because this shift pays $\$ 1$ more per hour in shift differential) and sleeps during the day may be judged as a "bad mom" for leaving her children with a caretaker overnight, or for not being able to see them off to school in the morning, or for sleeping

\footnotetext{
${ }^{17}$ Even mothers receiving State assistance for housing were under the expectation that found the best low-income community available.
} 
during the day when their child is awake. With Fathers often receiving praise for engaging in the same behaviors. For example, a Father who works the night shift because of the shift differential may be seen as smart and making a sacrifice for his family. Mothers in the work force may also need more flexibility in their schedule than an employee without children. For these reasons mothers often engage in low paying, lowskilled, part-time labor.

Motherhood can permeate mundane and seemingly unrelated aspects of women's lives. Women who become mothers often "institute regimes of diet, drink, and lifestyle" (Bailey 2002: 343). Mothers may find that the identity of 'good mom' restricts their diet in several ways. Mothers are expected to set a good example for their children when it comes to food. Intensive mothering ideology places the duties of teaching our children how to have healthy relationships with food primarily with mothers. In addition to being a good role model, mothers often restrict and change their diets to accommodate this greedy identity. Women who are pregnant restrict their diets regularly, nursing mothers also frequently regulate their food to ensure they are producing proper nutrition for their children. Mothers of toddlers frequently reduce the amount of spices in their own food in order to share with small children, or they may share significant portions of their own meals and limit their own caloric intake. All practices that become social expectations of motherhood.

When examined this way it is easy to see how the concept of doing and its relationship to motherhood becomes clearer. Being a good mom becomes "routinely fashioned in a variety of situations that seem conventionally expressive to begin with", such as middle-class mothers having disproportionate access to the resources necessary to 
fulfill the social expectations of being a 'good mom' (West and Zimmerman 1987:137).

This being said, the women in this study provided an interesting sample specifically because they are able to "accomplish" the identity of 'good mom' because they look the part.

\section{Looking the Part}

Research has shown that individuals work to manage the impressions others may have of them in an attempt to "construct more beneficial, less threatening, surroundings" (Schlenkler and Weigold 1992:134) and to highlight "facts about themselves that might otherwise not be apparent in the short interactions in which they normally engage" (Goffman 1959:30). It also may allow them to conceal an identity that they do not wish for others to know they possess. When an individual holds an identity as central, they are likely to engage in behavior that reinforces that identity to their self and to others (Snow and Anderson 1987). Self-presentation offers women with children the chance to appear to others as the mothers they would like to be and the ideal to which they aspire. Mothers use countless self-presentation tactics to claim their identities (Collett 2005):

"A woman who has yet to regain her prepregnancy figure may disclose to a stranger that she just had a baby, or a woman might explain the bags under her eyes are from staying up all night caring for her infant...a woman may conform to the opinions of other mothers in a playgroup, she may also use self-enhancement to advertise her strengths and admirable qualities, emphasizing her calm demeanor or discussing how she labored without the use of an epidural" (330). 
It is no doubt that self-presentation is important for women with children as they seek the approval of the title 'good mom' from countless significant others in their lives. Goffman (1959) theorizes that our "given impression", or "mask", represents our own personal conception of ourselves and the social role we are attempting to enact (19). In this way impression management can be an opportunity for mothers to not only demonstrate their attachment to the role of mother, but to also demonstrate their capacity to execute the responsibilities and the qualities expected of them, and to actively cultivate this image through their behaviors. It is their opportunity to contribute to the narrative of motherhood and to be active participants in their own biographies.

Snow and Anderson (1987) found that mothers engage in a wide variety of impression management behaviors:

“'Procurement or arrangement of physical settings and props' (mothers may convert their formal dining rooms into playrooms for the children); 'cosmetic face work or the arrangement of personal appearances' (mothers may purchase clothes befitting the role, whether the concern is comfort or modesty); 'selective association with other individuals or groups' (mothers may belong to playgroups or 'Mommy and Me' clubs); and 'verbal construction and assertion of personal identities' (mothers will often speak of themselves as a mother and accept opportunities to relay that information)"

In fact, most impression management literature focuses our attention on the way that social actors manage the impressions others have of them by directly altering their own behaviors in an effort to shape favorable meanings and interactions. It is common for mothers to engage in impression management strategies, such as managing their own and their children's appearances. According to Goffman (1959), appearances can be anything 
an onlooker can observe- clothing, grooming, habits, surroundings, props, and verbal and non-verbal actions.

The blog post at the beginning of the introduction provided an example of this. The mother authoring the post calls on upon multiple props to demonstrate her 'good mom-ness'. She begins with what is perhaps the biggest prop of all, the suburbs. As far as motherhood and family life go, you can't get a better symbol than suburbia. She goes on to discuss how her house is "tidy" again her domesticity becomes something she can put on display to signal her good mom-ness. She also refers to her attire as being an indication of her mom identity, "I wear skinny jeans and cardigans and flats, typical mom wear". She also called upon props in her purse that conveyed messages about the type of mother she was. "I carry my voter ID card, my passport, my library card, my driver's license (20 years behind the wheel and not a blemish on my record, knock on wood), and a bunch of Band-Aids and snacks because you never know where you'll find a kid, or fellow mom, in need". In other words, she is a participant in democracy, with middle-class ideas about and abilities to travel and experience the world, she cares about education and literacy, she is safe, she is prepared, and she is helpful. She is thrifty with her family's money and uses home hair dye and "drug store wrinkle cream”. She sounds like a great mom.

Every mother interviewed for this paper acknowledged that managing the mother identity may dictate changes to an individual's appearance; like carrying a diaper bag instead of a fashionable purse, trading in high-maintenance hairstyles for ponytails, exchanging business attire for athleisure wear or skinny jeans and cardigans, moving from their trendy city apartments to suburban homes, all the way down to trading in the 
fancy and fun ports car for a more practical minivan. (In the case of my moms that minivan likely has a sticker that reads, "Kids up in this Bitch" or "I USED TO BE COOL". An interesting observation here is that the mothers that held the idea that motherhood made them uncool were overwhelmingly mothers who become mothers early in adulthood.)

\section{"My mom uniform is always on"}

Many of the women interviewed made comments about their "mom uniform" and how their clothes were significantly less about self-expression of their individual identities as it was about looking the part. According to Bailey (2004) pregnant women who were first-time mothers reported that this phenomenon of looking "like a mom" begins for many women with their first purchase of maternity clothes. And while that may be in part to the limited selections available in maternity clothing, although in recent years that seems to be changing with fast fashion outlets like H \& M, Old Navy, and Target now carrying maternity lines, it is also the result of social interactions. By the time these women become mothers they "have been involved in enough interactions and accumulated sufficient previous information that they can effectively adopt and sustain the new status. An important part of implementing this social identity and maintaining it is looking the part" (Collett 2005, Cahill 1989, Goffman 1959).

Multiple mothers mentioned their "mom uniform" as a way to signal to others that they are 'good moms'. Melody, a 32-year-old mother of a 4-year-old and a 6-year-old in 
Vermont, stated that she was "a walking L.L. Bean ad" since she became the mom of two boys.

"How do people know I am a mom? Well to start, the flannels, thermals, fleece-lined leggings, 'puffy' vest or sweater, boots (usually my Ugg's) or flats in the warmer weather, a messy bun, a little make-up, a Yeti of coffee. I look the part. You know? Like I could take the kids for a hike and then make them a batch of warm oatmeal cookies between school and field hockey or boy scouts (laughing)...but seriously. I feel like my whole closet is just stuffed full of 'mom uniforms'. I don't own one sexy dress anymore. I used to have so many cute clothes. I even look like a mom when my husband and I go out for date night."

Her upper middle-class status is apparent in her statement but so is the cultural narrative available to women with children, like her. She discusses both the physical attire and the cultural meaning that it carries. The name brand clothes that indicated her class status and the style of her clothes that signaled 'boy mom' to others. She also points to the expected behaviors of having free time for her children to monopolize. The notion that she is available for hike or cookie baking after school reveal her status as stay-at-home mom. Her kids are also involved in scheduled extracurricular activities (i.e. field hockey and scouts), pointing to an investment in concerted cultivation (Lareau 2011).

Annette Lareau (2011) coined the term concerted cultivation in her pivotal work, Unequal Childhoods: Class, Race, and Family Life, where she argued that middle-class parents are more likely to engage in the normative model of parenting that tells parents that their children need structured activities and lessons, that parents are to be actively involved in the day-to-day lives of their children, and that it is the parents role to identify and nurture the talents and skills of their children. This parenting model is evident in 
Melody's comment above, in fact the comment reads almost like a "how-to-manual" in middle-class parenting. She is certainly hitting her cues (Goffman 1959).

The mom uniform is more than attire. It is more than a comfy pair of leggings, a long t-shirt, a boyfriend cardigan, and ballet flats. There can also be props in a mom's costume. For example, a large purse is often called a 'mom bag' because it gives moms the room to carry all of the other essential items needed to meet the criteria for 'good mom'. Amanda, the mother of a 4-year-old son, pointed to her 'mom bag' to signal her achieved status as a 'good mom'. She exclaimed, 'I am a 'good mom'. I am seriously the kind of mom you want around. I have a 'mom bag' that can save a fellow mom in a moment of crisis (smiling proudly). I carry a little bit of everything in there: baby wipes, hand sanitizer, band-aids, a bottle of water, a snack or two, extra socks, a couple matchbox cars, some stickers, a notepad and a crayon, and sunscreen."

\section{"My kids are clean and dressed to impress"}

The work of Collett (2005) draws our attention to another, although "indirect selfpresentation" that involves the use of associates for their own benefit. The work of Gillespie (1980) discusses practices such as those of first ladies who engage in their husbands' political self-presentation and suggests that the politician surrounds himself with symbols to suggest certain "necessary, yet intangible traits" (111). At times during a mother's impression management practice a child can be a "prop, only there for display, just one part of the mother's appearance. Infants make exceptional props for two reasons. 
First, young children are not viewed as complete persons and therefore not complete distractions (Goffman 1963:74). For example, a mother may engage her young child while in the middle of another conversation with adults and receive little if any social sanctioning. She may sooth a tired baby by swaying back and forth and hushing them as she engages in a conversation with a friend, without taking away from the intimacy of the conversation between the two adults (Goffman 1963:126).

Second, young children and infants are "open persons" (Goffman 1963:126). He theorizes them as exposed individuals who are open to be approached or engaged at any time. Social rules that restrict the behaviors of adult interactions do not apply to young children. For example, when adults meet it is highly unlikely that either person will pinch the cheek of the other or boop their nose, but this is relatively common behavior when interacting with young children. This treatment of young children illustrates how social practices such as these make children more comparable to objects than to other individuals.

The women who participated in this research reinforced these theories about mothers using their children as props in their impression management performances. Often this was done in implicit ways, with mothers simply posting pictures of their children or families when they were dressed well. Erin, a 35-year-old mother of a 2-yearold daughter is a good example of this strategy, "Pic cause baby girl is rocking her jumper and big bow today!!" or "My babies make me so proud" along with a picture of her 2 children dressed in matching velvet Christmas elf costumes. School picture day was another example of a time when it was apparent that this was a strategy that was used by many of the mothers in this research. This strategy was interesting in part because all 
mothers in the study reported using it to some degree, regardless of social class,

occupation, education, or racial background. This reinforced the cultural narrative that a 'good mom' is one with model children.

Bailey, a 33-years-old mother of three children under the age of 10 pointed to her children as the reason she was a 'good mom' in her own opinion. She stated, "I do believe I'm a good mom. My children are well taken care of. My home is clean. I work to make everything possible for my children. They are in clean clothes, they are clean, and they are educated. I think that the reasons I am a 'good mom' should make all moms 'good moms"'. Bailey was not alone in arguing that her claim to being a "good" parent was bolstered by the appearance of her children. Jennifer, a 24-year-old mother of two children under 3 years of age, is another example of how mothers internalize this social standard of appearances, both theirs' and their children's. During her interview she discussed the lengths she goes to in order to make sure her kids "look like 'good kids"'.

"I don't make a lot of money. I try really hard, but I don't make enough to buy my babies name brand clothes. I don't want them looking like little white-trash babies though. So, there are a couple things I do, cause I want people to know that they have a 'good mom' and that they are well taken care of. Like, in the Spring and Summer I go to a lot of garage sales in the really nice neighborhoods early Saturday morning and I buy bags of clothes. Name brand stuff. I get really nice stuff for them. Sometimes it even still has the tag. I like that cause then I know how much money I saved. Sometimes I joke it makes me a better mom than some rich lady who can just go buy it at the store. I had to go out and really look to find the deal I got. I mean I put A LOT of effort into making sure my babies look just as cute as those rich babies. I don't want them treated any different."

Another way that mothers may use their children as props in their own impression management practices may be highlighting their children when they are engaging in a 
praise generating activity. It is not important whom the praise is directed at either the mother or the children, because the mother can bask in the child's behavior as "a reflection of the mother herself and subsequently a part of the mother's own selfpresentation" (Collett 2005:331). The tendency to "judge the individual socially by the company he is seen in" means that children's actions, behaviors, accomplishments, and successes influence outsiders' perceptions of the adults associated with them (Goffman 1963:104).

This was illustrated regularly in the closed group. Mothers posted pictures of awards their children received in what were called \#ProudMaMaMoments and other mothers in the group would then give them virtual pats on the back, or Atta-Mama's. Often these activities were used not only to illustrate their children's successes or achievements but also to highlight their successful, even if sometime unconventional parenting. Lena's 4-year-old-daughter participates in traditional Irish dance classes and Lena posts many pictures of her dressed in adorable little dance costumes illustrating their success in introducing traditional parts of their Irish heritage into their daughter's life and fulfilling the cultural expectation that 'good' parents actively participate in cultivating their children into well-rounded, well-adjusted adults.

\section{Conclusion}

The overarching moral tale expressed in the interviews revolved around women's representation of themselves as good moms. They all sought to reshape or even undo a 
cultural script that made marijuana user into a greedy identity that threatened to erase their other identities, but most importantly threatened the coveted identity of good mom. For a few mothers in the study this sentiment almost took a tone of, "as long as you are trying to be a good mom, with what you got" as they worked to reshape the narrative in a way that was less restrictive and more accommodating to their everyday lives.

The mothers who participated told stories that highlighted a process in which they are consistently working to achieve this morally and socially valued identity while managing a gauntlet of personal and social obstacles along the way. They actively worked to create images of themselves that were reflections of the cultural narratives that they experienced and valued in their everyday lives. They were engaged in a "routine accomplishment embedded in everyday interactions" (West and Zimmerman 1987:126). Mothers discussed "playing a part" that came complete with a costume, props, lines, and queues.

When examining how women accomplish the identity of good mom several parallels to the way that Candace West and Don Zimmerman (1987) theorized that individuals accomplish gender. Their pivotal work sought to "propose an ethnomethodologically informed, and therefore distinctively sociological, understanding of gender as a routine, methodical, and recurring accomplishment"(126). This work envisions motherhood in a similar way. Motherhood theorized this way is "undertaken by women...whose competence as members of society is hostage to its production" "doing" the identity of good mom "involves a complex of socially guided perpetual, interactional, and micropolitical activities" (126). 
The women in the study who learned to perform the part of 'good mom' in relatively convincing ways reported that props played a vital role in their performances. Every part of their visible identity becomes a vital part of the meaning making process; the comfy and easy attire that falls into the category of "mom wear", the neighborhoods and homes they live in, the cars (SUV's and vans) they drive, the activities they engage in, the occupations they hold. All of these props shape the way that others perceive them as mothers. Reinforcing the idea that identity formation is a joint accomplishment that requires an internalization of socially accepted meanings as well as a recognition that the performance meets social standards before a social identity is bestowed upon a person.

Mothers relied on more than objects to convey their good mom identity to others. Children also regularly became props through which messages of good mothering were conveyed. The notion that "clean kids", "well-dressed kids", and "well-behaved kids" were signifiers of a woman's capacity to meet the social expectations attached to the identity of good mom was clear in the performances of these mothers. Children's achievements were also often used as props to demonstrate her expert "mom-ness". Goffman's (1959) work is useful in understanding these objects and even their children as props in their performance. The social meanings embedded in these objects and actions make her performance convincing and more than a speech act on her part. 


\section{CHAPTER SEVEN:}

\section{"I can be an amazing mom and be high": managing incompatible and greedy individual identities}

On the outside of this narrative of 'good moms' are women with children who engage in stigmatized behaviors, or carry stigmatized identities, and find themselves in vulnerable social positions as "Sancti-mommies" lurk around every corner waiting to pounce with their judgmental righteousness that empowers them to critique the mothering of other women. These self-appointed guardians of the 'good mom' identity become active defenders and reproducers of the cultural narrative that flattens women's identities and erases the experiences of their everyday lives.

As the narrative of being a "good mother" becomes more restricted and restrictive the identity itself becomes more and more untenable for larger numbers of women with children. It is for this reason that the 'good mom' identity is often a fragile one that must be managed daily, as it is easily disrupted leaving them open for social sanctioning from the sancti-mommies' constant policing, as well as, society at large. Public perceptions of what a "good mother" is or isn't become constant measures for these women during their daily negotiations. It is apparent that most mothers that participated in the interviews for this research project had a healthy internalization of the fact that the sanctimonious 'good mom' was always potentially just one post away. This alone can make the prospect of mothering a daunting and anxiety ridden task, add to that the ever further reaching power of the State to judge, regulate, and punish mothers and their activities and you have a 
social system that quite effectively regulates mothers everywhere, or at least the public identities that women with children perform.

For this reason, impression management becomes vital in helping women convey both competence and exceptionalism to self and their audience in a social setting where she desires nothing more than to be measured as successful, to belong to the good moms' club. Goffman (1959) calls the practice of "accentuating certain facts and concealing others" selective self-presentation or impression management (65). Impression management serves many purposes. It can help to "maintain a single definition of the situation and ensure smooth interaction" (Goffman 1959:255). It is also related to selfconcept (Gecas 1982), self-esteem (Brown, Collins, and Schmidt 1988), and self-beliefs.

That is not to say that all mothers experience this greedy identity to the same degree or in the same ways. Motherhood is a social, cultural, and political construct that takes on a variety of meanings in innumerable social contexts. For example, mothers of differently aged children experience their identities in much different ways, as do middle-class and poor mothers. In addition, mothers can often hold multiple identities, at times these identities are compatible with the identity of being a 'good mom' and require no impression management. These are what are considered low-cost social identities, while others may be considered high-cost, or greedy, as they come with severe social costs and, likely, require careful management. Goffman (1959) addressed this issue by stating,

\footnotetext{
"Some identity attributes carry low social costs because they are over axes of identity (such as a hobby or cultural taste) that are not viewed as directly tied to a political power or because they represent the unmarked pole on a politically or socially charged axis (such as heterosexual identity or white racial identity). ...identities not considered greedy because of
} 
their unmarked social value or lack of connection to salient disputes are less likely to be lifestyled than socially greedy ones."

It is for this lack of a universal experience that I make the argument that being a marijuana user can also be defined as a greedy identity for the mothers in this study. In the context of mothers who use marijuana, we can see a clear example of an identity that will "wash over" and pervade other aspects of the individual's social identity. That is not to say that there are social identities that are not disrupted or threatened by marijuana use, even in places where this usage might still be illegal, but 'good mom' certainly isn't one of them. For instance, a rock band member smoking a joint behind a bar he is preforming at does little to disrupt his social identity. In fact, it may reaffirm it for others. This is not the case for a woman with children who holds the socially prestigious and valued title of 'good mom'.

The mothers in this study actively engaged narratives that challenged the greedy identity created by the current cultural narrative that surrounds motherhood. They have worked as both individuals and as a collective in a concerted effort to create counternarratives about motherhood that challenged a variety of the prevailing cultural notions of mothers that possess these problematic, threatening, and greedy identities; such as working-mothers, single-mothers, poor mothers, and yes, marijuana using mothers. 


\section{Personal Counternarratives}

Individual level identity work or the "use of these signs, rules, and conventions by individuals to create images of themselves" (Schwalbe and Schrock 1996:115). Our experiences and identities as parents are shaped by multiple intersecting ideologies, social structures, and personal histories. Individual management of problematic or "stigma attracting" identities will be differentiated by a myriad of traits such as gender, marital status, family form, age, race, class, sexual orientation, mental disability, poverty, and reliance on social assistance programs. These traits are all important in establishing the power an individual has to avoid, resist, minimize, or challenge the stigmatization of a discrediting attribute in a given social interaction or social location (Collins 1999; Hays 2003; Lupton and Fenwick 2001; Phoenix and Wollett 1991).

In addition to individual experiences, cultural messages are constantly working to shape the meaning attached to specific social identities. 'Discourse' is a broad concept that is used to refer to different ways of constituting meaning specific to a particular group or culture. These discourses are tied to the construction of identities and practices associated with them (McGannon \& Smith 2015). It is true that the current discourses about motherhood and mothers circulate particular meanings which then "become forms of truth and difficult to challenge because they are also tied to gender ideologies (i.e., expected behaviors based on cultural values and norms). These practices include the prevailing notion that women's true calling is to have children and care for them" (Bailey 
2001:119). It is not uncommon for women with children to create personal counternarratives to the cultural narratives of motherhood. As they do they are actively engaged in the meaning making process in an effort to create cultural norms and expectations that are less restrictive and more in line with the lived experiences of their everyday lives.

There are multiple ways of speaking with and about ourselves available for use within the different cultural discourses that exist in a given social context. As mothers experience the material conditions of their social worlds they are able to identify and replicate the practices that they have come to attach to motherhood. Collett (2005) writes that the "running joke among parents is that, although one needs to take lessons and pass a test to demonstrate driving ability and earn a driver's license, anyone can walk into the hospital, give birth, and walk out with a baby" (327). Individuals are not given in full detail how to play the part or what conduct is required; rather, they are given "a few cues, hints, and stage directions" (Goffman 1959:72) about what makes a good mother. By the time women reach adulthood the vast majority of them have encountered and internalized the powerful gender discourse that shapes the practice and identities of mothers.

A growing body of research reveals that many mothers who have adopted this hegemonic understanding of motherhood find it to be isolating, frustrating, exhausting, and demanding in ways that leave them struggling to live what they find to be fulfilling lives (Crouch and Manderson 1993; Hays 1996; Lupton 2000; Stone 2007). Mothers who attempt to fulfill the social expectations of multiple social identities often find themselves feeling as though they are unable to live up to the high standards set by the ideology of intensive mothering or the ideology of the ideal worker (Stone 2007). 
This fits with the assessment of Zygmut Bauman (1996) as he argues that in the postmodern era people now seek to escape binding and restrictive identities. The restrictive narrative defining what is a good mom means that many women with children experience motherhood as a difficult and oppressive identity; one that requires an escape for many. As such, some of the mothers who struggled with the cultural narrative frequently engaged in the act of defining alternative practices and activities, this act can be seen as an attempt to fill the identity gap left by the hegemonic practices of "intensive mothering". Those who struggled and continued to internalize the narrative of intensive othering just found themselves falling short.

Those who fail to live up to the ideology of intensive mothering can find themselves the recipients of an ever-intensifying rhetoric about the consequences of failing in their role as mother. Leigh, a 37-year-old mother from Louisiana was the first to point to this fear of or acknowledgment of the potential for stigma "everyone is so quick to judge...society does seem to judge moms in general for a lot of things that are none of their business". With her comment she also acknowledges the effects of the greedy nature of the identity of motherhood. Motherhood, with its restrictive practices and oppressive rhetoric, has become a marked identity that influences how women enact and embody the identity of mother. 


\section{Holding a competing greedy identity- 'Marijuana-mom'}

'I believe what other people think a 'good mom' is and what I think a 'good mom' is, well... they're very different. Let's just say that. I mean, I can be an amazing mother and still smoke pot once a day. Maybe even more. I think the older generations don't believe women can be both a good mother and smoke marijuana. But I do. I do it every day. I am a great mom. My kids are loved. They have everything they need in life. We have a clean and safe home. They are clean and well-behaved. Nobody even knows that I am high. I don't see the problem. But I think there are quite a lot of people who truly think that I am not a good mother if I smoke. I also believe that there's probably a fair amount of older folks who I wouldn't even guess smoked themselves if they never told me! You can't win them all, but I KNOW I am a good mom regardless."

-Jess, 29-year-old married mom of a 2-year-old and a 4-year-old

Mothers who engage in drug use are easily seen as failing to fit the hegemonic model of a "good mother" (Banwell and Bammer 2006; Barnard and McKegancy 2004; Bays 1990; Broom 1994; Dodsworth 2012; Ettorre 2004), but how intensely they will experience social sanctions for embodying a competing greedy identity, like marijuanamom, is often out of their hands in several ways. As Bailey (1999) points out, an individual's ability to avoid social stigma is directly correlated to two factors, the amount of power their identities allow them hold and activate in the shaping of social discourses, and the power of the unmarked to control the cultural practices included in the discourse. In this work, I define marijuana-moms- as mothers who engage in daily marijuana use and also engage in the work of creating counternarratives, or discourses that challenge the understandings of motherhood in ways that include regular marijuana use (queue the pearl clutching sancti-mommies). 
On several occasions during my observations and participation in the online groups I was able to witness marijuana-moms actively shaping the narrative about mothers who use marijuana. During an interaction between five group members (two of which later became participants in this research project) the following conversation about whether or not you could be a good mom when you were 'high' took place.

OP (original poster) Kristin: Being present and sober seem like the cornerstones of good parenting... right? How can you parent if you are high?

Melissa: I can play Legos for like 6 hours if I smoke first, that makes my kids happy! I think that makes me a good parent, Kara.

OP/Kristin: Ha-ha. But don't you worry? Like what if your kid got sick or hurt and you were high? Could you be a good parent then?

Dawn: "I am really not trying to come off as condescending, so please do not take offense. It is clear that you are not a pot smoker. Being high isn't like being drunk. You can still function. Rather normally as a matter of fact. I use for both medical and recreational purposes and I don't stop taking my medicine just cause I have to be somewhere or take care of my kid. I use it to keep me from puking when I have migraines, which is often. I use it for the pain caused by the migraines. I also use it to sleep when my anxiety is bad. I don't usually smoke enough to be 'stoned' until after my kids go to bed, but if they wake up and I am stoned I can still take care of them, just fine. If the house caught on fire, I could and would save them. It is called a buzz kill, lol. I would no longer be high. That simple.

Adrenalin.

Third party: I use it for chronic pain. I am a better parent when I smoke marijuana than I am when I take all the meds the dr gave me.

OP/Kristin: So, you think you are just as good of a mom than if you were sober?

Melissa: Yes, it relaxes me and helps me get into the activities my children enjoy, from coloring to playing in the back yard. I am alone with my children many weekends, holidays, and nights and it's entertaining in a sense because I lack adult interaction. Sometimes it makes me more easily overwhelmed to be high, usually, though, it makes me more focused on my children and being present with them. Sober me has a hard time having fun playing tag or digging in the dirt.

Dawn: right? You said sober and present. I am ok with just present. As long as you aren't drunk. I think that is an issue. 
Third party \#2: I don't think it makes you a 'bad mom', unless your kids are going without. I mean if you are spending grocery money on pot that is a problem.

Melissa: totally an issue

OP/Kristin: Drunk is definitely an issue.

Third party \#2: Just do it after your kids go to sleep. That is what we do. My kids never see it. And I sleep better.

Third party: Or just messed up on pills. Some of them have way worse effects than pot. And they are addictive, Kara!

Dawn: Amen. I would rather smoke or eat an edible while parenting than take my migraine meds.

OP/Kristin: My name is KRISTIN.

-Summer, 2018.

The conversation above illustrates several of the recurring themes that were also present in the majority of the 53 interviews conducted for this paper. The recurring themes included: marijuana as “mommy's little helper", that it is better than the other alternatives, and that responsible use is key to maintaining her identity as a 'good mom'.

\section{“Mother's little helper"}

The most popular counternarrative that arose from the analysis of the interview data was that their use enhanced their ability to fulfill a number of social expectations that were embedded in the cultural narrative of what 'good moms' do. The counternarrative created challenged the cultural message so bluntly expressed by 'experts' and a group of other significant social actors that "marijuana and motherhood do not mix" (Bays 1990:882; Broom 1994; Harrington, et al. 1995; Emmett 1998; Hogan 
1998; Barnard 1999; Richter and Bammer 2000; Denton 2001; Jackson 2002; Barnard and McKegancy 2004; Ettorre 2004; Hogan et al. 2006).

My marijuana-moms rejected this dominant cultural message and engaged in the creation of personal counternarratives that claimed the contrary and will become vital to reconstruction of the collective identity of 'good moms'. For them, their regular marijuana use actually makes them better mothers. The mothers often attributed this to marijuana's ability to provide an escape from the stress, anxiety, and depression that mothers often report ${ }^{18}$ accompanies the restrictive identity of motherhood in the age of “intensive mothering” (Beck 1992; Bauman 1996). Cary was clear in her confirmation of this during her interview,

"There are days when marijuana absolutely makes me a better mom! There are days when I am just not fit for human consumption (laughing). Those days I put a little extra marijuana sugar in my coffee, and I am chill. I can roll with the day. I can be a better mom. But, aside from the effects of marijuana, the fact that I have used so much in my life and am familiar with it and the potential benefits and side effects, I can better educate my children on the uses and effects of marijuana and that makes me a better mom. My kids deserve a mom who is chill and not freaking out about life. So, yes. THIS (pointing at a coffee mug she has been sipping on throughout the interview) makes me a 'good mom',"

In fact, a large portion, twenty-three, of the forty respondents who reported that marijuana use was part of their everyday routines relied upon this "mother's little helper" counternarrative to some degree as they negotiated this troublesome part of their

\footnotetext{
${ }^{18}$ The CDC published a report in December of 2017 stating that women were twice as likely to experience lasting bouts of depression than men. Approximately 10-12\% of mothers in the US report experiencing depression in the first year of their child's life. Fathers had a much lower reporting rate with only $4 \%$ of fathers in the study reporting that they experienced depression in the first year of their child's life. Women were also three times more likely than men to point to domestic and parenting responsibilities as contributing to their depression.
} 
individual identity. Britney, a 32-year-old single-mom discussed how she activated this counternarrative during her interview when she stated, "I don't think it has an effect on my parenting, except that maybe it helps me get some better sleep so maybe on those days I am a little more patient”. For Britney, marijuana was something she did in the privacy of her own home after her children were in bed. It was the equivalent of have a glass of wine or two before going to bed ad a way to alleviate the stress of the day. "Being $a$ single-mom is hard and exhausting. But sometimes my brain just has a hard time shutting off all the stress of being a single-mom, of dealing with everything by myself. It is a few minutes of relaxation and hopefully a better night's sleep. I am a better mom if I am rested".

Several of the mothers pointed to marijuana's ability to relieve stress and to relax them during their interviews, highlighting the benefits to their ability to mother. Bailey, a 33-year-old mother of three children aged 10, 9, and 3 stressed this as her main motivation for use during the day in her interview, "At night it helps me sleep, but during the day I think it gives me more patience to be a better mom. I am way less irritable and more patient, for sure”. Bailey was not alone in this claim that it improved her ability to parent. Beth, a 34-year-old mother of two children aged 5 and 2, also used this strategy during her interview. "It can relax me and help me get into the activities my children enjoy, from coloring to playing in the back yard. I am alone with my children many weekends, holidays, and nights and it's entertaining in a sense since I lack adult interaction. Sometimes it makes me more easily overwhelmed to be high, usually, though, it makes me more focused on my children and being present with them". 
Beth's response calls upon a powerful cultural narrative about motherhood, as illustrated previously in Chapter 5, being a 'good mom' means being there or present for their children. Beth uses this important cultural narrative as the cornerstone of her personal counternarrative when she claims that marijuana enhances her ability to be present with her children. By the end of the interview analysis is was clear that this was common practice among marijuana-moms. A 38-year-old mother of a 1-year-old son named Kristy, activated this same personal counternarrative saying the "days that I spend hours on end with my toddler I find it helps me to stay in the moment and to be patient with him. I think that's really important for both of us." Kelly, a 34-year-old mother of a 4-year-old daughter also expressed that marijuana use enhanced her ability to be a 'good mom' but was not necessary for her to be one. "I am a great mom on or off marijuana. Sometimes I can really get down to her level and focus on something with her for longer if I am stoned. That makes her feel important."

In addition to enhancing their ability to be present and in the moment with their children, mothers also reported that marijuana made the tedious and mundane life of mothering more tolerable. Stone (2007) showed how the formerly high-powered career moms of her study would often pick up tasks that could fill the gap left in their identities when they head home full-time after having children. For the mother's in her study becoming the PTA president, organizing carpools, or engaging in local politics could all be strategies to demonstrate that they were 'good moms'.

Marijuana-moms like Katey, a single-mom of a 7-year-old, and Amanda, the mother of a 4-year-old in Iowa, pointed to marijuana's ability to enhance creativity as part of their personal counternarrative. "I feel cannabis sometimes gives me patience to 
do things I may find tedious. Such as watching the same movie or reading the same book over and over. I am more creative with my art ideas. But not necessary to be a good mom, just helps (laughing)”. Amanda's response was similar, “I think that it makes playing with play-doh and coloring much more tolerable. I am not really into pretend play and I find that it positively affects my ability to play and engage on that level".

This personal counternarrative positions marijuana as the new 'little yellow pill'. There is social, cultural, and political truth to this counternarrative. In 1966 The Rolling Stones released a song entitled "Mother's little helper", in which they make some powerful and extraordinarily relevant critiques of the cultural practices of motherhood.

"What a drag it is getting old"

“'Things are different today', I hear every mother say

Mother needs something today to calm her down

And though she's not really ill, there's a little yellow pill",

"She goes running for the shelter of her mother's little helper

And it helps her on her way, gets her through her busy day"

“' 'Things are different today', I hear every mother say

Cooking fresh food for a husband's just a drag

So, she buys an instant cake and burns a frozen steak"

"She goes running for the shelter of her mother's little helper And it helps her on her way, gets her through her busy day"

"Doctor, please, some more of these 
Outside the door, she took four more

What a drag it is getting old"

$$
\text { -Lyrics for "Mother's little helper" }
$$

I argue that for a growing number of women raising children in the age of legalization marijuana has become the new "little yellow pill". It provides mothers with an escape from the strain they experience in their everyday lives due to the unattainable cultural expectations placed on women with children to be 'good moms' and the material realities that structure and organize their lives in ways that prevent them achieving this socially valued identity (Jackson 2002; Ettorre 2004). Yesenia, a pregnant mother of two children aged 6 and 3, illustrated this parallel during her interview:

"I don't think it makes me a bad mom. Actually, I think that having a good time away from all the 'mom stuff', like barbies and diapers and crying miniature humans is good for moms. Well parents. It is a wonderful disconnect for a little while and that allows me to regroup and be a constant present parent. I feel this not necessarily only with marijuana, just in general, there are other ways to do this too, but marijuana is something you can do regularly. I don't think it is okay to drink every day. And I don't like drinking around my kids. I don't want to be that disconnected."

In addition to the mother's little helper counternarrative, Yesenia's statement indirectly points to another important counternarrative that was engaged by marijuanamoms during their interviews as they attempted to construct their marijuana use as nonthreatening to their coveted identity of 'good mom'. She explicitly points to marijuana's ability to enhance her ability to be a 'good mom' but she also implies that the available and more socially accepted alternatives may actually be more problematic than marijuana use. 


\section{"Better than the alternatives"}

During the interview transcript analysis I uncovered a second common counternarrative used by several of the mothers in which they position their marijuana use as being better than the alternatives. It quickly became apparent that this counternarrative had more than one implied meaning. In one sense it referred to the alternative as being sober, in general; in another sense this counternarrative serves the function of discrediting other legal and often more socially, culturally, and politically accepted alternatives to marijuana use (such as, alcohol or pharmaceuticals).

The first version of this counternarrative, refers to the alternative to being a marijuana-mom as being a sober mom, (i.e. a good mom). Allison, a 25-year-old singlemother of a 3-year old, and Laci, a 35-year-old mother of two children both relied heavily on this counternarrative in their interviews. Allison was quick to justify her marijuana use by saying, "I am calmer if I smoke. On days when I do not smoke my anxiety gets bad... Like I am a bad mom, and then that makes my anxiety worse (shaking head). I am kinder to myself when I smoke. And I think that makes me a better mom". Laci also justified her daily use during her interview by claiming, "It relaxes me better than anything else, really. I have some health issues and it really helps with chronic pain that makes me cranky some days. I am not a very good mom when I am in pain, but pain pills make me sleepy and then I really have a hard time being a 'good mom"”.

The second version of this counternarrative, where marijuana use is positioned as being better than the alternative refers to the alternative to marijuana use as drinking 
alcohol or consuming pharmaceuticals. It was apparent in Megan's interview that she drew heavily on this counternarrative. When asked if she thought her marijuana use affected her ability to parent her two sons, she responded:

"I honestly think using marijuana has helped me be a better mom to my boys. While I know that wine is the "publicly accepted" mom vice, I omit the hangovers, drunk behavior, and other health problems that come from an alcohol dependency. I have seen that side. My marijuana use has helped me be a more patient and thoughtful mom. Through my use I often reflect and become introspective about my parenting, I think that makes me a better mom. Plus, I have my mental faculties about me. I am in a place where I am not interested in things that do not allow me to grow as a mother and professional while using. I don't think that alcohol does that."

Megan, who is a single-mom working as a bartender to support herself and her children while she manages to make her way through a master's degree, attributes her attitude about the alternatives in part to a DUI that she received last year. "I had like two drinks at work before going home and next thing I know I am sitting in jail. I just couldn't stop thinking, ‘what kind of mother gets arrested for drunk driving?' I would have not been sitting in jail if I had smoked a joint before leaving work. Pot makes me a better parent". In addition to providing a clear demonstration of the better than the alternatives counternarrative, Megan's personal counternarrative demonstrates the importance of another powerful narrative "improving yourself as a mother". In this sense, improving yourself is better than the alternative of continuing to be a mediocre or less that great mother. This is indicative of other mothering practices like reading books and magazines on parenting. In Megan's case the knowledge she seeks about parenting and her ability to be a 'good mom' lie within her. For Megan and other mothers like her, marijuana use becomes a tool for self-improvement and not "just an escape from reality, 
like getting drunk. I don't know any moms who say, 'last night while I was drunk I had this life-changing realization', ya know?'”

For mothers like Megan and Jennifer, a 35-year-old mother of a 1-year-old marijuana becomes a 'mom hack' in their bag of tricks. They both pointed out multiple ways that they believed marijuana use to be a positive alternative to other more socially accepted practices for reducing stress, anxiety, and depression. The majority of mothers interviewed acknowledged at some point that alcohol and pharmaceuticals were the most common socially acceptable methods for mothers to reduce stress. In fact, they were often positioned as being more socially acceptable than healthier alternatives for stress reduction, like exercise or recreational sport participation (McGannon and Schinke 2013; McGannon et al. 2018).

Jennifer stated in her interview that she used marijuana for "Anxiety reduction" and highlighted the benefits of marijuana use like "waking up without a hangover, being more relaxed at bed time" claiming this "results in better rest and happier parents in the morning". She goes on to say "I sometimes think it makes me a better mom. I am more relaxed and more easily get down on my kiddo's level and act silly with her and have fun!"’.

\section{"Responsible use is the key"}

"I don't feel it affects your ability to be a good mom unless you're over using it and spending money on marijuana instead of other responsibilities."

-Jess, a 29-year-old mother of two in Vermont 
The final counternarrative that emerged from the interview data was that of responsibility being key. This counternarrative, was similar to the counternarrative of mother's little helper, in that it was able to draw upon the power of an already existing cultural narrative about what it means to be 'good moms'. Mothers were able to demonstrate their ability to be a responsible mother and a marijuana-mom. The individual narratives of the marijuana-moms became a pool of collective meaning from which a collective identity could be pulled. The counternarrative that being responsible was key to the maintenance of the 'good mom' identity was constructed as a defense to social stigma that could be applied to the collective identity of marijuana-mom. This alternative construction of motherhood stressed being responsible to the extent of being selfless, much like the contemporary cultural construction of good mothers.

The contemporary cultural construction of 'good moms' is one that sets the social expectation where women are expected to shed potentially conflicting or damaging social identities when they become mothers. This loss of personal identity in exchange for a greedy social identity can be difficult and is frequently seen as an act of self-sacrifice (Stone 2007). Many of the mothers interviewed pulled heavily on this cultural narrative to solidify their membership in the collective of 'good moms'.

Being selfless is a powerful cultural message used to construct our beliefs about mothers. The narrative is attached to an array of practices, behaviors, and attitudes that mothers may hold. In the instance of marijuana use, Leigh, a 37-year-old mother of two children in Louisiana, a state that has legal Medical Marijuana, discussed the ways being a mom affects her identity of marijuana user. Leigh claimed at the very beginning of her 
interview that she had been a regular marijuana user since she was a young teenager and that the behavior has continued consistently for her entire life. She used this as an opportunity to point out how becoming a mother has changed her usage. "Now I only smoke when I am home. I use a vape pen, so my kids don't ever see flower. I keep it from pretty much everyone now, if they knew they might not think I'm as good of a mom?"

Amanda, a mother of a 4-year-old in Iowa also made it clear that part of the counternarrative she used to maintain her identity as 'good mom' despite her consistent longtime marijuana use was one of being responsible. She is a professor at a college in a conservative rural area. This social setting creates what she feels is a need for greater secrecy about her relationship with marijuana. During her interview she pointed to the threat of social stigma and her responsibility as a good mother to protect herself and her family from that stigma as an important part of why she actively positioned herself as a 'good mom', even if she is high.

"I don't talk about my use unless I know someone is sympathetic. I almost never share that I smoked while pregnant with my son due to the stigma attached to that practice. I also only smoke at home and always in a private room to minimize contact and the smell in case someone pops in. We just moved recently to a small town and these folks are likely to knock on your door for any reason. We try not to broadcast it in case others stigmatize it considering we are trying to integrate ourselves into this new place. I think others might reconsider the stereotype surrounding usage considering that I basically debunk it. I am a motivated, successful and gainfully employed user which goes against the belief that it is detrimental to achievement, motivation and success."

- Amanda, 39 married Professor in Iowa

This was not an uncommon practice for other mothers in the study as well. There seemed to be an overwhelming desire to keep their marijuana use private, "now that I am 
a mom”. Only two mothers interviewed reported keeping her marijuana use private for a reason other than fear of stigma because they are also mothers. Both of these women reported that occupational factors were the primary cause they were secretive about their use.

In this way being responsible meant "keeping it quiet". This was more than just not using or talking about it in public settings. It also meant taking precautions to ensure that there were not formal sanctions placed on them for involvement. Katey, stressed the importance of following the laws surrounding marijuana use strictly in her own life, "I try to keep my marijuana use legal in regard to: I don't want to be pulled over and found high. Or trying to partake somewhere that forces others to walk through the smell. I try to be aware of who is affected by my use at all times". Similar to Katey's personal counternarrative was that of Pamela, a recently separated mother of two children also stressed this in her interview, “I don't smoke around others. I smoke at home only. I keep paraphernalia put away. I don’t discuss my use. My ex knows but I really need him to believe I don’t anymore, so I keep it very hush hush".

Kelly, a 34-year-old married mother of a 4-year-old who lives in North Carolina had a personal counternarrative that emphasized not just the need to "keep it quiet" from law enforcement or others that may not be intimate or close relations, but for Kelly there is also the santi-mommies, and the conservative community in which she lives to consider, "I only do it at home now, or with family members or close friends at gettogethers. Well I live in the bible belt so I am pretty sure I would have to wear a scarlet letter if the majority of the population in my staunchly republican tiny little town knew that I used marijuana”. 
Kelly's need to hide her use seems justified given the legal status of marijuana in North Carolina. As of 2018 marijuana had been decriminalized in North Carolina meaning it was not considered legal and there was no legal access. However, Katey and Pamela both live in states where marijuana use is fully legal. Maine and Vermont have passed legislation allowing anyone in the State over the age of 21 access to legal recreational and medical marijuana. This raises an interesting facet of the identity 'good mom' and the informal social pressures to be a 'good mom' as well as where they are coming from. Although for many of the mothers who said their first 'line of defense' was to keep their marijuana use secret it became quickly apparent that the people they spent most of time actively working to keep use from were their children and other family members, not the public or law enforcement. While mothers like Katey and Pamela were certainly working to not violate laws openly, they both admitted they placed more attention on maintaining their identity in informal social contexts, such as their own homes.

"We keep it on a high shelf in our bedroom just so the kids can't reach it. I don't think they've even seen it. We only consume at night in our home so I don't think anyone would even know unless we offered the information. But I don't feel the need to hide it. I don't think anyone would be surprised by my relationship with marijuana. If it concerned them then they likely are not 'my people'."

-Ellen, 39-year-old partnered with a 2-year-old in Vermont

There were also women with children who expressed that they engaged in little or no identity management practices as a result of their marijuana use. For example, Mal a 28-year-old mother from Iowa who proclaimed loudly during her interview, "I don't really care what people think. I know who I am. My kid thinks I am good mom. That is 
what matters". Mal's statement was the most direct in expressing that she has little fear of the social stigma that may accompany others' gaining knowledge about her relationship with marijuana. Briana, a married mother of two children in Oregon expressed that her fear of social stigma was muted in comparison to mothers like Pamela. Briana stated, "I don't take special precautions to hide it, but I don't tell the world either. I honestly don't care if people have issues with it because the only people's opinions I care about already know. It is legal in Oregon, so I see no issue with it. If ever it starts to affect my ability to be the best parent I can be I would stop immediately. There is no addiction to marijuana, at least not for me". Lena, a 38-year-old mother of two children in Oregon also reported engaging in few if any practices to hide her use, "When I use marijuana since becoming a mom, it is always in a safe environment for my family (like when the kids are asleep) and in small amounts". All of these mothers stress how they are responsible with their use and that is key to maintaining their good mom identity.

\section{Conclusion}

The counternarratives revealed during the analysis of the interview data become vital in understanding how marijuana-moms came to understand themselves as good moms, despite their conflicting and greedy identity of marijuana user. Mothers frequently relied on counternarratives like mother's little helper, better than the alternatives, and responsibility is key in an effort to normalize their stigmatized behavior. These mothers created counternarratives that pulled upon existing cultural narratives about what it meant 
to be a good mom. Often these counternarratives sought to weave positive social meanings attached to motherhood (i.e. selflessness/self-sacrificing, present, accepting, and supportive) with a currently problematic social practice (i.e. marijuana use while being a mother) in attempt to change the texture of their lives. The goal is two-fold, to lessen stigma and to move mothers from flattened cultural stereotypes to complicated and nuanced social actors.

The marijuana mom's social world may contain a variety of significant others that play a role in shaping her identity. They may include her child(ren), her immediate family, her extended family, those who make up her child(ren)'s networks of association (ex. Friends, healthcare providers, teachers, childcare providers), her employer, and the legal system. Every one of these social actors will play a role in the social identity that results from the negotiation these mothers will engage in to protect their coveted identity of 'good mom'. "In an ideal world where there is no social cost to an identity it is probably the case that most people would choose to be integrators of most of their identities. Commuting and lifestyling, while by no means caused solely by oppression, do tend to be higher for anyone identifying where there are social costs to living an identity openly and around others who don't share in the identity" (Brekhus 2003: 45). Marijuana using moms encounter and negotiate competing discourses both inside and outside of their social circles, in some ways the competing discourses enable these women to play an active role in reconceptualizing themselves while they also limit them (Bailey 1999). 


\section{CHAPTER EIGHT:}

\section{The groupwork of redefining the collective identity of "good}

\section{$\underline{\text { mom" }^{\prime \prime}}$}

Earlier chapters focused on the individual identity work that many of the marijuana-moms engaged in order to create personal identity narratives, while the following chapter will focus on the communal identity work. This is what Schwalbe and Schrock (1996) refer to as "the creation of identities as widely understood signs with a set of rules and conventions for their use" that is central to how mothers who actively engage in marijuana use position themselves as 'good moms' (115). It is for this reason that part of the focus of this research was to examine the repertoire of counternarratives that these women with children draw upon to create a new collective identity that reconstructs marijuana-moms as good moms, instead of "bad moms who don't deserve to be mothers at all" ${ }^{\prime 19}$.

The role the internet and online interactions play in our individual and collective identity constructions has been a topic of investigation over the last three decades, approximately. The majority of that research focused on how identities are constructed through interactions in anonymous online settings (i.e. chat rooms, online 'bars', and other open forums), however with the proliferation of social media sites like Facebook

\footnotetext{
${ }^{19} \mathrm{Kim}$, a 40-year-old mother said this about how significant others in her life had expressed they felt about mothers who smoked marijuana. Kim gave this as one of the reasons that she kept her use private and why she also limited her use until her children were in bed and never consumed in front of them.
} 
more attention needs to be paid to how social media sites that do not provide anonymous identities for individuals contribute to the individual and collective identity construction processes (Zhao et al. 2008). Facebook is consider a "nonymous" online space because it requires users to reveal their real names, has an optional place to display a photo or representation of their selves, and includes an "about me" section that users can use to weave a narrative about the self they hope to project. Recent research has explored how public and open social media spaces, such as Facebook, impact the actions of those participating. The importance of the findings of this body of research is its ability to indicate "that the online world is not monolithic, and online self-presentations varied according to the nature of the settings" (1817).

Identity is an important part of the self-concept, or the "totality of a person's thoughts and feelings in reference to oneself as an object. It is the part of the self by which we are known to others" (Rosenberg 1986). The construction of an identity is a public process, one that occurs through face-to-face interactions and virtual interactions in today's technologically advancing society. The proliferation of internet has changed the traditional conditions of identity production by removing the "corporeal body...it becomes possible for individuals to interact with one another on the Internet in fully disembodied text mode that reveals nothing about their physical characteristics" (Zhao et al. 2008: 1817). As their online, disembodied selves allow them to "play-act at being someone else" or to create identities that differ from their "real life" identities (Stone 1996).

Online identity construction can be an empowering process as it allows individuals to remove undesirable characteristics from our 'virtual self' it also allows 
individuals to express their "hidden selves" playing an important role in identity empowerment and construction (Suler 2002).However, it is important to remain cognizant of the ways that online environments like Facebook are not entirely anonymous, in fact, most people's Facebook identity is one that is constructed in full view of "anchored relationships"; such as family members, neighbors, colleagues, and offline acquaintances. That is not to say that an online acquaintance can't also constitute an "anchored relationship" (Zhao et al. 2008). Many of the mother's interviewed revealed that their interactions in these closed online groups were grounded in anchored relationships, they reported that they thought of the other mothers who were members of these closed online groups as friends. As a result, the women who participated in the interviews for this research were likely to get to know each other through online interactions or mutual friendships that encouraged interaction. The concept of anchored online relationships will be important to understanding the collective identity construction of good moms.

Identity construction in an entirely anonymous online setting enables individuals to feel free to be whomever or whatever they want. Anchored relationships in those settings, however, can remove anonymity and place constraints on the freedom of identity claims made by individuals. Although, this does not remove the need for selfpresentation, or self-performances (Zhao et al. 2008; Stone 1996; Suler 2002). The online mother's groups that were the source of the majority of the participants in this study, "The Cage" and "Misfit mothers" were groups full of anchored relationships, these were relationships anchored in online interactions. Although a few of the mothers were 
acquainted in 'real life' many of the anchored relationships within the group did not know each other offline.

This familiarity does not mean that identity management is not occurring. Selective self-performances continue to occur in places where individuals are fully identifiable, but in such contexts they are constrained and tend to conform to established norms. As the number of connections, a person has within an online group increase and become more intense the level of conformity with group ideals also increases (Brennan and Pettit 2004). While it is true that anonymous social spaces allow more freedom to express new and multiple identities, it is not the case that these identities are unlimited or unconstrained. Zhao et al. (2008) argues that "the nonymous online world, emerges as a(n)...environment where people may tend to express what have been called the 'hopedfor possible selves"” and anchored relationships can limit the ways that women constructed the "hoped-for possible selves" (1819).

These projections of self are interesting to our understanding of how the construction of a greedy identity, such as good mom. It is worth noting that the social setting of a closed, or private, group dictates that "the hoped-for possible selves users projected on Facebook" would be different from the identities they projected in face-toface interactions among people they know or those formed in anonymous online groups. The Facebook selves these mothers were constructing appeared to be decidedly socially desirable identities that these women with children aspire to have offline but have not yet been able to embody for one reason or another (Zhao et al 2008:1830). There appear to be some unique features associated with the way in which mothers used closed Facebook groups to construct their "hoped-for possible selves." 
Many of the women did not have public Facebook profiles so it was not possible to examine the narrative that they used to construct their 'hoped-for possible selves'. Several women did have public pages making it possible to see their identity construction through their online profile. I was also 'Facebook friends' with four of the women who answered the recruitment posts in the closed online groups. These friendships created anchored relationships between the participants and myself that may have impacted their self-performance during the interview process, as they had given me access to personal narratives that they may not have shared within the closed group. This access to her public performance may have limited or constrained the performances available to her.

\section{Selective self-presentation}

Sarah, the mother of two teenagers who lived in a small Midwestern town provided a prime example of this. During her interview Sarah disclosed that her oldest child had recently been caught abusing Adderall, a powerful stimulant often easily available through prescription to teens and college-aged individuals who claim to have trouble focusing while studying. After examining Sarah's activity on her public social media page, it became clear that her "public identity" as a 'good mom' was being carefully crafted in ways that might cover "the cracks" in her mask (Goffman 1959). Her "newsfeed", a stream of posts in chronological order or a history of activity, was a beautiful construction of a "good mom with good kids". 
The steady display of countless pictures taken from the stands as she cheers on her son as he plays on the Varsity Basketball team as a Sophomore combined with dozens of posts containing inspirational quotes about loving your family are mixed in with a dozen or more pictures of her daughter holding medals that she has won for being in debate competitions and pictures of the home she and her husband recently built alongside a small lake. A quick scroll through her newsfeed illustrates her selected selfpresentation. The narrative she weaves will tell you that she is a 'good mom'. The kind of mom who fulfills the standards set out for 'good moms' everywhere. She has clean, active, intelligent, seemingly well-adjusted teenagers. She clearly displays that she is active and present in their lives as she sits in the stands, and publicly celebrates their accomplishments as if they are also her own.

Over the months I spent participating in the closed online group I was able to observe many of the mothers engage in similar activities and narratives within the group. It was not uncommon for mothers to post pictures of their children/family engaged in activities that demonstrated their capacity to live up to the social expectation. Lisa, a 40year-old married mother of a 5-year-old also frequently posted pictures of her daughter's accomplishments as \#proudmamamoments, a hash tag often used as a "brag tag" for mothers to indicate that they were making a post that indicated that they had been 'good moms" as evidenced by her "good kids". This brings our attention to the way that "Facebook is a multi-audience identity production site. The control users have over the privacy settings of their accounts enables them to partition their Facebook pages into many 'back' and 'front' regions (Goffman 1959), whereby staging different identity shows for different audiences" (Zhao et al. 2008:1832). 
This does not mean that all of the mothers in the group were successful in crafting their desired or hoped-for possible self. Many of the women that participated in the online groups would also often fail at their selective self-presentation while engaging in dialogue within the group. Through observations it became apparent that as mothers became more integrated into the online groups they also became more likely to "let the cracks show" (Goffman 1959). As women engaged in the group and gained anchoring relationships with other members of the group they also seemed to become less restrictive with what facets of their lives they shared within the group. Their selective self-presentation became less selective. Since the groups were "by invitation only" every member had at least one anchoring relationship, and the majority of those relationships were face-to face. The number of online anchor relationships a person gained through participation in the group relied on several factors. For example, how likely the mothers were to 'friend' people on their personal pages that they did not know 'in-person', how long they had been members of the group, how active they were in dialogues, and how often they were the originator of posts.

Joni, a single mother of 10-year-old twins with severe autism, was a regular participant in the group who had gained several online anchoring relationships to the group through her interactions there. Her face-to-face anchoring relationship was a friend that actually had recently moved across the country. Joni often reported having few 'real life friends' and expressed her gratitude for the friendships she had formed through the mom's group. Her increased significant relationships obviously impacted the intimacy of Joni's interactions in the group. Her posts and responses soon became increasingly intimate and revealing of the 'cracks in her mask' (Goffman 1959). 
This became evident one night when Joni, in the midst of a fight with her husband as they were separating, appeared to swallow pills with alcohol while smoking a joint in a 'Facebook live' video that she posted to the closed group page. While many would assume that Joni would have been judged as failing to live up to the cultural expectations attached to motherhood that was not the case. Instead, mothers in the group rallied, found her anchoring relationship and made contact to check her welfare and get her much needed help. Joni entered counseling and used the online group as a place to discuss the “process of getting better for her kids' sake”.

This incident became a watershed moment in the group, after mothers witnessed the overwhelming support that Joni, a now known recreational marijuana user received in her moment of transparency. After this incident more and more mothers began to 'show their cracks' during their interactions within the groups, as well. The majority of the mothers did not shatter their masks in the same way as Joni, but it was clear after this incident that many of the mothers felt comfortable enough now to reveal their statuses as marijuana-moms, as well. The incident with Joni also became a central part of the identity construction process that began, with many mothers calling upon it as a way to help construct their own counternarratives of marijuana-moms as 'good moms'.

\section{“The Cage”: Online groups as identity generating spaces}

Bauman (2001) theorized that as society advances farther into modernity individuals will "seek safety" in communities. As the internet becomes more widely 
accessible and available to a growing number of people it also becomes a space where communities form. It is for this reason that I sought out and chose online communities like the two selected for this study. My goal was to find online groups that claimed to be 'communities' and stressed the importance of belonging. The two online communities selected were centered on the idea of motherhood as a practice that is difficult and requires support. These particular groups were created with the goal of providing mothers a virtual community to share their experiences and learn from the experiences of other "not-so-traditional" or "misfit" moms. Brekhus (2003) calls these types of identity communities "marked identity spaces" and theorizes that these spaces would provide mothers who do not identify with the hegemonic model of motherhood "a social space where one's marked identity does not have to be muted and toned down to meet mainstream conventions" (45-46).

Many of the marijuana-moms interviewed discussed these groups as being "sanctuaries for mothers", "safe spaces", and "happy places" where they felt free to be themselves and express their struggles with managing motherhood and their other identities; threatening identities in particular.

"Facebook enables the users to present themselves in ways that reasonably bypass physical 'gating obstacles' and create the hoped-for possible selves they are unable to establish in the offline world. Such 'digital selves' are real, and they can serve to enhance the users' overall self-image and identity claims and quite possibly increase their chances to connect in the offline world" (Zhao et al. 2008:1832).

In these closed online groups, many of the mothers discussed how they perceived a variety of statuses they held as being a threat to their identities as good moms and how 
their interactions in these 'marked identity spaces' had profound impacts on how they now constructed their self-image and self-performance. This supports the findings put forth by other researchers, that these two worlds are more connected than previously theorized (Zhao et al. 2008 and Suler 2002).

"It is incorrect to think that the online world and the offline world are two separate worlds, and whatever people do online 'hold little consequence' for lives offline. In the Internet era, the social world includes both online and offline environments, and important skill people need to learn is how to coordinate their behaviors in these two realms." (Zhao et al. 2008:1831)

Collective identity construction in online 'marked identity spaces' becomes important in understanding not just how individuals work collectively to challenge cultural narratives that they find restrictive or unattainable due to structural or personal obstacles. This new understanding challenges 'the distinction between 'real selves' and 'virtual selves' or 'true selves' and 'false selves'. 'Virtual selves' commonly refers to online selves and 'real selves' to offline selves, but, as has been shown here, Facebook identities are clearly real in the sense that they have real consequences for the lives of the individuals who construct them." (Zhao et al. 2008).

Joni's story was just one example of a mother's 'virtual self' having real consequences for her 'real self'. Cora, is another mother who let her "cracks show" in her virtual self and found their to be consequences for her real self. She also had an incident in the group that distinctly altered her self-presentation in the group and one she claims led to changes in her real life, as well. This conversation between Cora, a 34-year-old mother of three who had recently expressed she was having trouble in her marriage: 
Cora (OP): Warning long post ahead:

I am not even sure why I am writing this here. I guess I just really need a place to vent. Do you all remember how I have been saying things with my husband have just been off lately? Well ladies I have figured it out and I am heartbroken, and mad, and sad, and so many things right now. Please help me make sense of this before I go crazy. I NEVER look at my husband's phone or his FB account but because he has been so weird lately I had a suspicion that there was something going on. So last night while he was asleep I got on his phone and looked through it. He has been sending some chick he works with all these flirty texts and liking all her pictures on FB. He no longer has his marital status public so people can't see it. From the texts it doesn't sound like anything physical has ever happened between them but it still feels like cheating. I am so mad at him. I do everything for this man. I mean everything. I try so hard to be a good wife and a good mom and this is how he treats me while I am here at home taking care of his family. I would never do this. Not that it is even an option for me. I am always busy being a mom. I look like a mom. No one hits on moms! Even if they did I would never feel like I could do that to my kids. Please tell me that other moms have been through this.

Jaci: Girl, kick his ass to the curb. You do not need this shit. You are a good mom to those babies. You will be a better mom without him causing you stress.

Joni: So sorry. I have not been through this but my husband and I did just separate and I can tell you that I am a much better mom now that he is gone. One less person I have to take care of. So I have more time for my kids.

Cora: How did you manage having to work and still find time to be a good mom?

Jaci: I just know that if I am not there at the house it is because I am at work trying to make their lives better. It is about them and giving them more. I think that means we are good moms, Cora. Good moms do what they need to do to make sure that their kids have good lives.

Cora: You all are right. He does stress me out all the time and I do take that out on my kids by being irritable or sad and not playing with them. Maybe I would be a better mom. You ladies are the best.

Cora's story is another instance where we see attempts to shift the collective narrative about what it means to be a good mother. Here the women in the group help her to shift the narrative that she draws upon in order to shape the meaning of 'good mom' and her 
own self-image. She opens the conversations by clearly drawing on the traditional narratives that surround motherhood. Narratives that tell us that good mothers are selfless, loyal to their families, good wives, who often lack any type of sexuality outside of their marriage. Cora has internalized all of these conventions of motherhood, however, after other mothers affirm that this narrative is outdated or incorrect she is quick to reject it and even show her acceptance of this new counternarrative that these mothers construct in front of her.

\section{"Finding your Tribe": Belonging and meaning making}

The sense of belonging that the women who join these groups repeatedly report feeling becomes important in understanding how collective identity construction becomes a valuable contributor to our personal identities. In her influential work, longing and belonging: parents, children, and consumer culture, Allison Pugh (2009) argues that to feel a sense of belonging means to have a desire to fit-in with a group or to feel as though your identity work you have displayed that you hold similar beliefs and engage in similar practices (27-35). The self-image that these women had of themselves as 'good moms' despite carrying what Goffman (1963) referred to as spoiled identities. In the case of my marijuana-moms, they sought out others mothers who carried potentially troublesome identity statuses, much like their own. "Finding a group that was full of good moms who were also covered in tattoos, or unmarried, or marijuana users was huge for me. It was the first time I didn't feel judged for they way I was a mom”, says Dawn. 
Dawn, 39-year-old mother of two toddlers, stressed the need for belonging in her interview.

"The cage has been amazing for me. I don't really have any friends that I see face-to-face anymore. I mean it. I am not exaggerating. All of my best friends live in a different state at this point in my life. So, the cage gives us a place where we can still hang out everyday online. Sure, we are hanging out with 700 other moms, but they are cool, for the most part. We still have our own inside jokes, but we also have jokes that are just for cage members. We are like a little clique on Facebook. I spend way more time in the cage than I do on my own page. These are my people."

Amanda, a 39-year-old mother from Iowa, had a similar sentiment about her group membership and its importance to how she saw her self as a mother. "The cage is my tribe. They are not like all those judgey moms I encounter in my real life because of where I live mostly. These women get me. They are all cool enough to say, 'you don't have to be that mom"'. For mothers like Amanda, the most important part of feeling like she belonged was the lack of judgement she had felt throughout her experiences in the closed online group. Acceptance, or the desire for it, was a key part of the reasons given by most of the mothers for feeling as if they belonged in the closed groups.

Acceptance did not necessarily mean a lack of criticism from group members, in fact, it was quite common for mothers in the group to challenge the practices of other mothers. But for my marijuana-moms these challenges were often presented in constructive ways that allowed them to contribute to an alternative definition of 'good moms'. Because of the culture of acceptance that is clear in the rules of group membership, “Don't be a $C^{* * *}$, Kara” mothers were careful to pose their objections in less judgmental ways than they might had the interaction occurred in their face-to-face interactions or in a more public forum. I came to consider this as a part of their own 
selective self-presentation. In this case they may have kept their true feelings muted or completely out of the interactions for fear that if they appeared too judgmental in their comments other group members or the volunteer 'admins' might sanction them in some way. A member can be blocked, banned, or removed from the group for a short period of time at the admins discretion.

Megan, a 29-year-old mother of two boys, discussed the desire for acceptance and belonging and how it had impacted her interactions in the group. "I am usually way more blunt with people than I am in the cage. I mean I still express what I think I need to, but in the cage I try to not be judgey. One I don't want banned. It would be like being grounded from my cool friends. Two, I am really trying hard to be a better person.". In Megan's comment we see how the internalization of the rules of group membership have become an important part of how she manages her interactions.

While rules about etiquette are vague, there is an underlying assumption within the group that no one is perfect and that is okay. The mothers make efforts to open, understanding, and supportive of the other women in the group. However, this is not always the case for these mothers outside of these closed online spaces. It is still common for women negotiating motherhood and marijuana use in today's world to be vulnerable to experiencing guilt in relation to the cultural discourses that suggest 'good moms' prioritize their children above all else financially, emotionally, and in every other way. Something that was demonstrated by the mothers who relied on the responsibility is key counternarrative to restore their identities as 'good moms'. 


\section{Conclusion}

During the time I spent doing ethnographic observations it became very apparent that identity construction is not an act in isolation. It is a social process. This coincides with Becker's (1953) research on why people did or did not engage in marihuana use. He too argued that it is

"the result of a sequence of social experiences during which the person acquires a conception of the meaning of the behavior, and perceptions and judgements of objects and situations, all of which make the activity possible and desirable. Thus, the motivation or disposition to engage in the activity is built up in the course of learning to engage in it and does not antedate this learning process. For such a view it is not necessary to identify those 'traits' which 'cause' the behavior. Instead, the problem becomes one of describing the set of changes in the person's conception of the activity and the experience it provides for him" (Becker 1953:235).

This project also placed significant focus on how the women who were interviewed conceptualized their marijuana use and how they experienced it as mothers. The majority of the marijuana-moms interviewed for this study met Becker's assessment, "the most frequent pattern of use might be termed 'recreational'.... a relatively casual kind of behavior in comparison with that connected with the use of addicting drugs". In fact, all but two of the mothers interviewed reported their use to be recreational or "for pleasure". This designation was intended to demonstrate the "noncompulsive and casual character of the behavior" (Becker 1953:235). Both of the other mothers reported daily marijuana use for medical purposes; one reporting use for chronic pain caused by arthritis and the other reporting use to control severe social anxiety. 
Since this identity of marijuana-mom was one they took up voluntarily it seemed to add to its greedy nature. It the two mothers who reported medicinal use also reported that they felt very little stigma or pressure to change their behaviors surrounding their marijuana use. However, mothers who reported recreational use also described shared strategies for keeping this part of their self a 'hidden self'(Suler 2002). They engaged in behaviors such as only using at home, or not talking about their use in front of others, they made efforts to keep it secret. Many of the mothers only discussed their use in the closed online groups were they felt it was safe to disclose without causing damage to their identities as 'good moms'.

The exclusivity of these online spaces allowed women to feel safe to divulge this part of their identity. Together women engaged the process of redefining what it meant to be a 'good mom' in a way that included those who used marijuana on a regular basis. This space became fertile ground for creating new meanings about what it meant to be both a 'good mom' and a daily marijuana user. These women were able to demonstrate that these two greedy identities were not mutually exclusive of one another. You could, in fact, be both. The more this message was reaffirmed for them within this 'marked identity space' the more comfortable they became embodying both identities. 


\section{CHAPTER NINE:}

\section{Conclusion}

The women who participated in this research engaged in daily identity construction while participating in closed or private online 'marked identity spaces' and revealed some important findings with important implications for future research on identity construction as well as how people experience and negotiate competing greedy identities. Marijuana-moms were an ideal group to help us to understand this aspect of constructing your personal identity and the counternarratives that become useful in shifting the cultural meaning attached to greedy identities. These marijuana-moms used an online community to construct a counternarrative that combined a set of culturally established practices and narratives about motherhood that solidified them as 'good moms' despite their marijuana use.

The practices that these mothers used in their self-performance were part of a larger set of practices that had been established and accepted by society as the practices of 'good moms'. They often included a mixture of compulsory tasks that many of the women admitted they begrudgingly engaged in due to social expectations and not out of personal preference. These practices included everything from where they lived to what they ate and wore. All of these practices were essential to her efforts to look the part. This illustrated not only their personal internalization of the existing cultural narratives about 'good moms' but also her ability to use this narrative to construct a cultural counternarrative that included her. 
They acknowledged that 'good moms' were moms that looked the part in other ways, as well. The most common way that marijuana-moms claimed this privileged identity was through their own appearance and the appearances' of their children. Many of the women referred to their look as a 'mom uniform' and described a generic aesthetic that sent a universal message that she was, in fact, a mother and likely a good one. They described a few variations of this uniform, but all contained comfortable and conservative choices for their mom uniform that complimented most body types with minor adjustments. This assured that most mothers could comfortably signal their status as a 'good mom' to others with a few key articles of clothing.

Marijuana-moms were able to pull on contemporary cultural narratives about being a 'good mom' that they had already internalized through their experiences with motherhood in an effort to create counternarratives that normalize their marijuana use and manage the greedy nature the identity marijuana-mom carries. In order to resituate themselves as 'good moms' in the eyes of other mothers that they see as embodying the title and in the eyes of significant others that inhabit their social worlds they worked as individuals and as a collective to reconstruct the identity of 'good mom' in less restrictive and more inclusive ways. The cache of cultural narratives available to pull from included standard cultural expectations like 'good moms' are selfless and self-sacrificing, they are present and sober, loving and accepting, and always there to help and support their children.

Many of the mothers pulled on these contemporary narratives of motherhood in the process of constructing the new counternarratives of themselves as 'good moms' despite their status as marijuana-moms. Mothers interviewed told narratives about their 
everyday lives that constructed marijuana as the new "little yellow pill", also known as mother's little helper. This was perhaps the most popular of all the counternarratives. Perhaps because of the pop culture relevance that makes it an easy narrative to follow. Mothers frequently discussed marijuana as enhancing their abilities to live up to several of the key characteristics from above. Moms reported that marijuana increased their ability to be present, loving, and supportive.

Marijuana-moms also went to great efforts to position their marijuana use as "better than the alternatives" of sobriety, pharmaceuticals, and alcohol. Discussing how these all create states in them that they saw as undesirable for mothers to be in. Moms that called "being high" a better alternative to sobriety did so for two reasons. The first came from the two mothers who reported using for medicinal purposes, claiming that usage made them less irritable, relieved pain or stress an allowed them to be better mothers as a result. The second was more common among recreational users. They claimed that their marijuana use actually enhanced their abilities to be 'good moms'. By making the mundane tasks of mothering more pleasurable it made them better moms because they were more willing to engage in imaginary play; such as Legos. Play-doh, and coloring. It gave them the ability to be there/present with their children and that made them better moms.

The last narrative that mothers activated to protect their identity as a 'good mom' was a counternarrative that called upon the contemporary cultural narrative that mothers should be responsible for their children, their homes, and to some extent their marriages, This counternarrative focused on the ways that 'responsibility is the key' to being a 'good mom'. In many ways this counternarrative was created to be a sort of universal dismissal 
of critiques of behavior. It holds the message that if a mother is engaging in an activity that may threaten her status as a model mother as long as she does this responsibly then her status as a 'good mom' is less threatened. For example, if a mom wants to spend an evening out drinking with friends that is fine and not a threat to her identity as long as she takes measures to ensure that her children will be taken care of by a sober and responsible person. Responsibility is the key!

By pulling on narratives that highlight characteristics of motherhood that have been established and accepted as the "proper" way to perform the role, marijuana-moms are actively engaging other mothers in an effort to reshape the meanings attached to their particular patterns of parenting. They are creating new counternarratives that rely on socially established norms of mothering to demonstrate that the use of marijuana does not preclude a mother from the title of 'good mom'. These mothers construct a new image of mothers that includes marijuana use in the same way that some mothers include wine use in their narratives of motherhood.

\section{Changing cultural meanings one post at a time}

In the age of legalization marijuana-moms are engaging in a collective effort to reshape the meanings attached to motherhood as well as marijuana use. They have used closed or private online groups that provided safe spaces to challenge cultural norms of motherhood. In these "marked identity spaces' they worked collectively to create counternarratives that positioned these mothers as 'good moms' despite a behavior 
that would typically discredit them making the coveted status nothing more than a pipedream. However, with each new post or comment made in support of the behavior we see this stigma associated with the behavior lessen, and more support for the counternarrative that a mother who uses marijuana daily can be a good, loving, and attentive mother. The resistance to marijuana using moms claims of being 'good moms' forces them to confront and negotiate an identity within a sociocultural space bounded by their claims to both a mainstream identity and a marginalized identity. The value of listening to these women's voices is the value of their testimony and experiences, which have been systematically ignored and dismissed.

\section{Significance}

The findings of this study have many important implications for the understanding of identity construction. The results suggest that identity is not an individual characteristic; it is not something innate in a person. Rather it is a social product, the outcome of a given social environment and hence performed differently in varying contexts. The social characteristics of the environment in which they find themselves will affect how "individuals will choose to claim identities that help them better situate within the given social environment. 'True selves,' 'real selves,' and 'hoped-for possible selves' are products of different situations rather than characteristics of different individuals.

This research is important to the current discussion being had regarding marijuana use and mothering. It is important in broadening the focus of the discussion from that of 
"deviant" drug-using mothers to one that allows marijuana using mothers to be situated alongside other "good" mothers. The goal is to contribute to a greater body of feminist literature that challenges society to not evaluate these women as 'good moms' or "bad moms" based on one characteristic, behavior, or identity. Rather we should strive to create a more nuanced understanding of marijuana using moms and their everyday lived experiences within the growing climate of legalization. This is vital in challenging the notion that one aspect of a person's life should erase or override so many other aspects.

This research offers and alternative perspective of mothers who engage in druguse. By looking at mothers who use marijuana, a drug that is increasingly being decriminalized and whose use is increasingly becoming more accepted in mainstream culture, we can challenge existing narratives that frame mothers who use drugs as "unfit" or "bad" moms. By examining the narratives used by mothers who use marijuana to maintain their identities as "good mothers" we can see how they are working to reshape the cultural narrative surrounding motherhood.

Instead of following other researchers in positioning these mothers as deviant or apologetic in their behavior this research illustrates the ways in which these mothers maintain the morally valued identity of mother and how their mothering practices express both challenges and acceptances of culturally normative views of mothering. By challenging the cultural narratives that work to split mothers into "good" and "bad" they are able to reconstruct motherhood through acceptance of new practices and understandings of what it means to be a mother. To attempt this without developing a greater understanding of the lived experiences of these women would surely result in a superficial understanding of motherhood. 
This research might also be useful in discussions regarding depression in mothers. As the cultural ideal of what it is to be a good mother becomes increasingly more unattainable for mothers outside of the hegemonic understanding of what a 'good mom' is, so increases the "sense of individual failure if the ideal is not lived up to" (Dodsworth 2012: 101). By focusing on women who fit into the hegemonic ideal of a 'good mother' in many ways despite their use of marijuana we can uncover powerful narratives that contradict the cultural assumption that drug-using mothers are dangers to their children and create a more complex understanding of mothers who use drugs.

\section{Implications for future research}

This could have important implications for the well-being of mothers and their children as states negotiate this new terrain of legalized cannabis use. Historically, drug use by mothers has been cause for imprisonment and the removal of her children from the home, often landing them in foster care. Drug-use by mothers has also been a factor in excluding them and their children from access to social services, such as food stamps and child care assistance. If drug use in itself was no longer seen as sufficient cause to define a woman and a "bad mom" these resources would still be available and provide many of these mothers' avenues to "ameliorate some of the gender inequities in child-rearing and domestic work" (Banwell and Bammer 2006) and reduce the marginalization of "other mothers" within the hegemonic discourse of mainstream mothering. Placing the narratives of drug-using mothers alongside those of other mothers allows us to illustrate 
the ways these mothers strive to position themselves as "good mothers". And in doing so, shift the cultural focus from individual mothers and toward the cultural and structural conditions of mothering in an effort to remove and improve upon many of the cultural contradictions experienced by all mothers, irrespective of drug use, social class, or social position. 


\section{MATERIALS APPENDIX:}

Demographics survey

\section{Self-complete portion:}

Please answer the following questions to the best of your knowledge. Remember all answers are kept strictly confidential. Identifying characteristics will not be used in project findings and all names will be changed to ensure anonymity.

Marital status:

Race:

Age:

Number of children and ages:

Estimated Annual Household Income:

Employment status:

Occupation:

Education level:

What is the status of Marijuana in the state in which you reside? Criminalized legal medical legal recreational

How would you describe your use?

Rare Occasional Frequent Daily 
Tell me about an average day for you.

Who do you think of when you think of a "good mom?

What is it about this person that makes you think they are a "good mom"?

Where do you think you got your ideas about what it means to be a "good mom"?

Do you think that you are a "good mom"?

What is it about you that makes you think this?

Do you believe that your understanding of what it means to be a "good mom" and what society tells women it means to be a "good mom" are similar/different? Why?

Tell me your opinion on marijuana, it's use, it's legalization, etc.

Describe your relationship with marijuana. How does it fit into your life?

Did you use marijuana before becoming a mother? How often? How did you consume the drug? Describe your usage patterns.

Have you used marijuana since you became a mom? How often? How do you consume the drug? Describe your usage patterns.

How do you feel marijuana affects your ability to be a "good mom"? Why?

Do other people you are close to use marijuana? Who (relationship to you)?

How open are you with others about your relationship with marijuana? Who do you share this information with? Who do you exclude from it? Why?

What behaviors, if any, do you engage in to keep your marijuana use private?

What do you think others would think of you if they knew of your relationship with marijuana and/or use? 


\section{REFERENCES:}

Arendell, Terry. 2000. "Conceiving and Investigating Motherhood: The Decades of Scholarship". Journal of Marriage and the Family. 33:689-695.

Bailey, Lucy. 1999. "Refracted Selves? A Study of Changes in Self-identity in the Transition to Motherhood". Sociology. 33(2):335-352.

Bailey, Lucy. 2001. "Gender shows: First Time Mothers and Embodied Selves. Gender \& Society. 15: 110-129.

Banwell, Cathy and Gabrielle Bammer. 2006. "Maternal habits: Narratives of mothering, Social position and drug use". International Journal of Drug Policy. 17:504-513.

Barnard, Marina. 1999. "Forbidden Questions: Drug-dependent parents and the welfare of their children". Editorial. Addiction. 94(8):1110-1111.

Barnard, Marina and Neil McKegancy. 2004. "The impact of parental problem drug use on children: What is the problem and what can be done to help". Addiction. 99:552-559.

Bauman, Zigmut. 1996. "From Pilgrim to Tourist- or a Short History of Identity". 18-36 in Stuart Hall and P. Du Gay (eds.). Questions of Cultural Identity. London. Sage.

Bays, Jan. 1990. "Substance Abuse and Child Abuse: Impact of Addiction on the Child". Pediatric Clinics of North America. 37(4):881-904.

Beck, Ulrich .1992. Risk Society: Towards a New Modernity. London. Sage.

Becker, Howard S. 1953. "Becoming a Marihuana User". The American Journal of Sociology. 59(3):235-242.

Blair-Loy, Mary. 2003. Competing devotions: Career and family among women executives. Cambridge, MA. Harvard University Press.

Blumer, Herbert. 1969. Symbolic Interactionism. Englewood Cliffs, NJ. Prentice-Hall.

Bock, J.D. 2000. "Doing the thing right?: Single mothers by choice and the struggle for legitimacy". Gender \& Society. 14:62-86.

Boulton, Mary Georgina. 1983. On being a mother: A study of women with preschool children. London and New York. Tavistock.

Breen, Courtney, Amanda Roxburgh, and Louisa Degenhardt. 2005. "Gender Differences among Regular injecting drug users in Sydney, Australia 1996-2003". Drug and Alcohol Review. 24:353-358. 
Brekhus, Wayne. 2003. Peacocks, Chameleons, Centaurs: Gay Suburbia and the Grammar of Social Identity. Chicago and London. University of Chicago Press.

Brennan, Garrett and Paul Pettit. 2004. "Esteem, identifiability, and the internet. Analyse \& Kritik. 26:139-157.

Broom, Dorothy H. 1994. Double Bind: Women affected by alcohol and other drugs. Sydney. Allen and Unwin.

Brown, Stephanie, Rhonda Small, and Judith Lumley. 1997. "Being a 'good mother'". Journal of Reproductive and Infant Psychology. 15:185-200.

Butler, Judith. 1990. Gender Trouble: Feminism and the Subversion of Identity. New York, NY. Routledge.

Cahill, Spencer. 1989. "Fashioning Males and Females: Appearance Managements and the Social Reproduction of Gender”. Symbolic Interaction. 12(2):281-298.

Charmaz, Kathy. 2001. "Qualitative Interviewing and the Grounded Theory Analysis". The Handbook of Interview Research: Context and Method. Eds. Jaber F. Gubrium and James A. Holstein. Pp. 675-694. Thousand Oaks, CA. Sage.

Chesley, Noelle. 2011. "Stay-at-home Fathers and Breadwinning Mothers: Gender, Couple Dynamics, and Social Change”. Gender \& Society. 25:642-664.

Collett, Jessica L. 2005. "What Kind of Mother Am I? Impression Management and the Social Construction of Motherhood". Symbolic Interactionism. 28(3): 327-347.

Collins, Patricia Hill. 1994. "Shifting the Center: Race, class, and feminist theorizing about motherhood". In E. Nakano Glenn, G. Chang, \& L.R. Forcey (eds). Mothering: Ideology, experience, and agency. Pg 45-91. New York, NY. Routledge.

Collins, Patricia Hill. 2005. "The meaning of motherhood in Black culture and Black mother-Daughter relationships". In M. Baca Zinn, P. Hondagneu-Sotelo, and M.A. Messner (eds). Gender through the prism of difference. Third ed. Pg 285295. New York, NY. Oxford University Press.

Crouch, Mira and Lenore Manderson. 1993. New motherhood: Cultural and personal transitions in the 1980's. Camberwell, Victoria. Gordon \& Breach Science Publishers.

Denton, Barbara. 2001. Dealing: Women in the drug economy. Sydney. UNSW Press. 
DeVault, Marjorie. 1999. "Comfort and Struggle: Emotion work in family life". Annals of the American Academy of Political and Social Science. 561:52-63.

Dodsworth, Jane. 2012. "Sex worker and mother: managing dual and threatening identities". Child and Family Social Work. 19: 99-108.

Douglas, Susan J. and Meredith Michaels. 2004. The Mommy Myth: The Idealization of Motherhood and How It Has Undermined Women. New York, NY. Free Press.

Edin, Kathryn and Maria Kefalas. 2005. Promises I Can Keep: Why Poor Women Put Motherhood Before Marriage. Los Angeles, CA. University of California Press.

Elliott, Sinikka; Rachel Powell and Joslyn Brenton. 2015. "Being a Good Mom: LowIncome, Black Single-Mothers Negotiate Intensive Mothering". Journal of Family Issues. 36(3):351-370.

Ellis-Sloan, K. 2013. "Teenage mothers, stigma and their 'presentations of self"'. Sociological Research Online. 19:9.

Emmett, G.A. 1998. "What happened to the "crack babies"”. Drug Policy Analysis Bulletin. 4:1-3.

Ennis, L.R. (Ed). 2014. Intensive mothering and the cultural contradictions of modern mothering. Bradford, Ont. Demeter Press.

Ettorre, Elizabeth. 2004. "Revisioning women and drug use: gender sensitivity, embodiment, and reducing harm". International Journal of Drug Policy. 15:327335 .

Eyer, Diane. 1996. Motherguilt: How our Culture Blames Mothers for What's Wrong with Society. New York, NY. Random House.

Gekas, Viktor. 1982. "The Self-Concept”. Annual Review of Sociology. 8:1-33.

Gekas, Viktor and Michael L. Schwalbe. 1983. "Beyond the Looking Glass Self: Social Structure and Efficacy Based Self-Esteem”. Social Psychology Quarterly. 46:7788.

Giddens, Anthony. 1991. Modernity and Self-Identity: Self and Society in the Late Modern Age. Cambridge. Polity Press.

Giddens, Anthony. 1992. The Transformation of Intimacy: Sexuality, Love, and Eroticism in Modern Societies. Cambridge. Polity Press.

Gillespie, Joanna. 1980. "The Phenomenon of the Public Wife: An Exercise in Goffman's Impression Management”. Symbolic Interaction. 3(2):109-126. 
Goffman, Erving. 1959. The Presentation of Self in Everyday Life. New York, NY. Doubleday.

Goffman, Erving. 1963. Stigma: Notes on the management of spoiled identity. Englewood Cliffs, NJ. Prentice-Hall, Inc.

Haines-Saah, Rebecca J., Joy L. Johnson, Robin Repta, Aleck Ostry, Mary Lynn Young, Jeannie Shoveller, Richard Sawatzky, Lorraine Greaves \& Pamela A. Ratner. 2014. The privileged normalization of marijuana use - an analysis of Canadian newspaper reporting, 1997-2007. Critical Public Health. 24:1. 47-61

Harrington, D., Dobowitz, H., Black, M., and Binder, A.. 1995. "Maternal substance use and neglectful parenting: Relations with children's development". Journal of Clinical Child Psychology. 24(3):258-263.

Hays, Sharon. 1996. The Cultural Contradictions of Motherhood. New Haven, CT. Yale University Press.

Hays, Sharon. 2003. Flat broke with children: Women in the age of welfare reform. New York, NY. Oxford University Press.

Hey, Valerie. 2004. "Perverse Pleasures- Identity Work and the Paradoxes of Greedy Institutions". Journal of International Women's Studies. 5(3): 33-43.

Hogan, D. 1998. "Annotation: The psychological development and welfare of children of opiate and cocaine users: Review and research needs". Journal of Child Psychology and Psychiatry. 39(5):609-620.

Hogan, Tina M. Smarsh, Barbara J. Myers, and R. K. Elswick Jr. 2006. "Child abuse Potential among mothers of substance-exposed and nonexposed infants and toddlers". Child Abuse \& Neglect. 30:145-156.

Jackson, M. 2002. Mothering and drugs: Four case studies. In H. Klee, M. Jackson, \& S. Lewis (eds). Drug misuse and motherhood. London and New York. Routledge.

Lareau, Annette. 2011. Unequal Childhoods: Class, Race, and Family Life. Berkeley and Los Angeles. University of California Press.

Link, B.G. and J.C. Phelan. 2001. "Conceptualizing Stigma”. Annual Review of Sociology. 27:363-385.

Lorber, Judith. 1993. "Believing IS Seeing: Biology as Ideology". Gender \& Society. 7: $568-581$.

Lorber, Judith. 1998. Gender inequality: Feminist Theories and Politics. Los Angeles, CA. Roxbury. 
Lupton, Deborah. 2000. “'A love/hate relationship': The ideals and experiences of firsttime mothers". Journal of Sociology. 36(1):50-63.

Lupton, Deborah and Jennifer Fenwick. 2001. "'They've forgotten that I'm the mum": Constructing and Practising motherhood in special care nurseries". Social Science and Medicine. 53:1011-1021

McCormack, K. 2005. Stratified reproduction and poor women's resistance. Gender \& Society. 19:660-679.

McGannon, Kerry R., Jenny McMahon, and Christine A. Gonsalves. 2018. “Juggling motherhood and sport: A qualitative study of the negotiation of competitive recreational athlete mother identities". Psychology of Sport \& Exercise. 36: 4149.

McGannon, Kerry R. and Robert J. Schinke. 2013. "My first choice is to work out at work; then I don't feel guilty about my kids": A discursive psychological analysis of motherhood and physical activity participation. Psychology of Sport and Exercise. 14: 179-188.

McQuillen, Julia, Arthur L. Greil, Karina M. Shreffler, and Veronica Tichenor. 2008. "The Importance of Motherhood Among Women in the Contemporary United States". Gender \& Society. 22(4): 477-496.

Mears, Ashley. 2011. Pricing Beauty: The Making of a Fashion Model. University of California Press. Berkeley and Los Angeles, CA.

Miller, P. 2001. "A critical view of the harm minimization ideology in Australia". Critical Public Health. 11(2): 167-178.

Moore, Dinty. 1995. The Emperor's Virtual Clothes: The Naked Truth about Internet Culture. Chapel Hill, NC. Algonquin.

Oakley, Anne. 1979. Becoming a Mother. Oxford. Martin Robertson.

Phoenix, Ann and Anne Woollett. 1991. "Motherhood: Social construction, politics, and psychology". In Motherhood: Meanings, practices, and ideologies. Newbury Park. Sage Publications.

Reinharz, Shulamit and Susan E. Chase. 2001. "Interviewing Women". CH 11 in Handbook of Interview Research: Context and Method. Eds. Jaber F. Gubrium and James A. Holstein. Pg. 221-238. Thousand Oaks, CA. Sage.

Rich, Adrienne. 1977. Of Woman Born: Motherhood as Experience and Institution. London. Virago. 
Richter, K.P. and Gabrielle Bammer. 2000. "A hierarchy of strategies heroin-using mothers Employ to reduce harm to their children". Journal of Substance Abuse Treatment. 19:403-413.

Riessman, C.K. 2000. "Stigma and everyday resistance practices: Childless women in South India". Gender and Society. 14:111-135.

Riley, D. 1983. War in the Nursery: Theories of the Child and Mother. London. Virago.

Roberts, Dorothy. 1999. "Welfare's ban on poor motherhood". In G. Mink (Ed). Whose welfare?.Pg 152-170. Ithaca, NY. Cornell University Press.

Rosenberg, M. 1986. Conceiving the self. Malabar, Fl. Robert E. Krieger.

Schwalbe, Michael and Douglas Mason-Schrock. 1996. "Identity Work as Group Process". Advances in Group Processes. 13: 113-147.

Schlenkler, Barry and Michael Weigold. 1992. "Interpersonal Processes Involving Impression Regulation and Management". Annual Review of Psychology. 43:133168.

Snow, David and Leon Anderson. 1987. "Identity Work Among the Homeless: The Verbal Construction and Avowal of Personal Identities". American Journal of Sociology. 92:1336-1371.

Stone, A. 1996. The War of Desire and Technology at the Close of the Mechanical Age. Cambridge, MA. MIT Press.

Stone, Pamela. 2007. Opting Out: Why women really quit careers and head home. Berkeley and Los Angeles, CA. University of California Press.

Suler. John. 2002. "Identity Management in Cyberspace”. Journal of Applied Psychanalytic Studies. 4(4):455-459.

Tardy, Rebecca. 2001. “'But I Am a Good Mom': The Social Construction of Motherhood through Health- Care Conversations." Journal of Contemporary Ethnography. 28:433-473.

Turkle, S. 1995. Life on the screen: Identity in the age of the Internet. New York, NY. Simon \& Schuster.

Warrior, Betsy. 1986. Housework: Slavery or Labor of Love. Fourth Ed. Betsy Warrior Publishing. 
Web Staff. April 17, 2017. "Study: Majority of Americans who say they use marijuana are parents". Retrieved April 20, 2018 (http://kdvr.com/2017/04/17/studymajority-of-americans-who-say-they-use-marijuana-are-parents/)

Weiss, Robert S. 1994. Learning From Strangers: The Art and Method of Qualitative Interview Studies. The Free Press. New York, NY.

West, Candace and Don Zimmerman. 1987. "Doing Gender”. Gender \& Society. $1(2): 125-151$.

Wiegers, Wanda A. and Dorothy Chunn. 2015. "Stigma and resistance: The social experience of choosing sole motherhood in Canada 1965-2010". Women's Studies International Forum. 51:42-55.

Williams, C. 1991. Case studies and the sociology of gender. In J. Feagin, A. Orum \& G. Sjoberg (Eds), A case for the case study (224-243). Chapel Hill: University of North Carolina Press.

Wilson, $\mathrm{H}$ and Huntington, A. 2006. "Deviant (m)others: The construction of teenage motherhood in contemporary discourse. Journal of Social Policy. 35:59-76.

Whitley, R and L.J. Kirmayer. 2008. "Perceived stigmatization of young mothers: An exploratory study of psychological and social experience". Social Science and Medicine. 66:339-348.

Worrall, Anne. 1990. Offending Women: Female lawbreakers and the Criminal Justice system. London and New York. Routledge.

Young, K. 1989. "Narrative Embodiments: Enclaves of the Self in the Realm of Medicine". In J. Shotter and K. Gergen (eds). Texts of Identity. Pp 152-165. Newbury Park, CA. Sage

Zartler, U. 2014. "How to deal with moral tales: Constructions and Strategies of singleParent families". Journal of Marriage and Family. 76:604-619.

Zhao, Shanyang, Sherri Grasmuck, and Jason Martin. 2008. "Identity construction on Facebook:Digital empowerment in anchored relationships". Computers in Human Behavior. 24:1816-1836. 
Jennifer Wilson was born in Coffeyville, KS although the majority of her childhood was spent in Charleston, SC before her family return to the Midwest in 1996. She was the first in her family to attend college when she earned an Athletic Scholarship at Independence Community College and her associate degree in 1999. Jennifer continued her education at Pittsburg State University earning her bachelor's degree in Sociology, with Academic Honors in 2008. Upon graduation Jennifer was accepted to the PhD program in the Sociology department at the University of Missouri-Columbia.

At Mizzou, Jennifer earned her master's and Doctorate degrees in Sociology with a Minor in Women's and Gender Studies. She was an active member in the Coalition for Graduate Workers, the Sociology Graduate Student Group, and the Women's and Gender Studies Club. Jennifer was also an active member of the community where she served as the President of a Women's amateur sports league for six years and engaged in many activities focused on empowering women to be active in their communities.

Jennifer is currently an Assistant Professor of Sociology at her Alma Mater, Independence Community College in Independence, KS, where she maintains her research interests in a variety of issues that relate to gender inequality and institutional culture. 